\title{
Functional capacity, physical activity and muscle strength assessment of individuals with non-small cell lung cancer: a systematic review of instruments and their measurement properties
}

\author{
Catherine L Granger ${ }^{1,2^{*}}$, Christine F McDonald ${ }^{2,3}$, Selina M Parry ${ }^{1,4}$, Cristino C Oliveira ${ }^{1}$ and Linda Denehy ${ }^{1,2}$
}

\begin{abstract}
Background: The measurement properties of instruments used to assess functional capacity, physical activity and muscle strength in participants with non-small cell lung cancer (NSCLC) have not been systematically reviewed.

Method: Objectives: To identify outcome measures used to assess these outcomes in participants with NSCLC; and to evaluate, synthesise and compare the measurement properties of the outcome measures identified. Data Sources: A systematic review of articles using electronic databases MEDLINE (1950-2012), CINAHL (1982-2012), EMBASE (1980-2012), Cochrane Library (2012), Expanded Academic ASAP (1994-2012), Health Collection Informit (1995-2012) and PEDRO (1999-2012). Additional studies were identified by searching personal files and cross referencing. Eligibility Criteria for Study Selection: Search one: studies which assessed functional capacity, physical activity or muscle strength in participants with NSCLC using non-laboratory objective tests were included. Search two: studies which evaluated a measurement property (inter- or intra-rater reliability; measurement error; criterion or construct validity; or responsiveness) in NSCLC for one of the outcome measures identified in search one. Studies published in English from 1980 were eligible. Data Extraction and Methodological Quality Assessment: data collection form was developed and data extracted. Methodological quality of studies was assessed by two independent reviewers using the 4-point COSMIN checklist.
\end{abstract}

Results: Thirteen outcome measures were identified. Thirty-one studies evaluating measurement properties of the outcome measures in participants with NSCLC were included. Functional capacity was assessed using the six- and twelve-minute walk tests; incremental- and endurance-shuttle walk tests; and the stair-climbing test. Criterion validity for three of these measures was established in NSCLC but not the reliability or responsiveness. Physical activity was measured using accelerometers and pedometers. Only the construct validity for accelerometers and pedometers was reported. Muscle strength was measured using hand-held dynamometry, hand-grip dynamometry, manual muscle test, one-repetition maximum and the chair-stand test, however only two studies reported reliability and measurement error and one study reported construct validity.

Conclusion: Currently there is a gap in the literature regarding the measurement properties of commonly used outcome measures in NSCLC participants, particularly reliability, measurement error and responsiveness. Further research needs to be conducted to determine the most suitable outcome measures for use in trials involving NSCLC participants.

Keywords: NSCLC, Functional capacity, Strength, Physical activity, Measurement properties, Systematic review

\footnotetext{
* Correspondence: catherine.granger@mh.org.au

'Department of Physiotherapy, School of Health Sciences, The University of Melbourne, Melbourne, Victoria, Australia

${ }^{2}$ Institute for Breathing and Sleep, Melbourne, Victoria, Australia

Full list of author information is available at the end of the article
} 


\section{Background}

Non-small cell lung cancer (NSCLC) is associated with significant disease burden, impaired physical status and diminished physical activity [1,2]. Due to the disease and treatment (surgery, chemotherapy and or radiotherapy) adverse physiological and psychological effects are prevalent in NSCLC, particularly exercise intolerance, weakness and impaired gas exchange and commonly a cycle of functional decline ensues [1]. Increasingly exercise interventions targeted at preventing the functional decline associated with NSCLC or improving the physical status prior to or after cancer treatment are the focus of research trials [3]. Three commonly used endpoints are functional capacity "the maximal capacity of an individual to perform aerobic work or maximal oxygen consumption" [4]; physical activity "any bodily movement produced by skeletal muscles that results in energy expenditure" [5]; and muscle strength "the maximum voluntary force or torque brought to bear on the environment under a given set of test conditions" [6]. The gold standard instruments (outcome measures) to assess these outcomes are laboratory based, which are not always feasible for use in research or clinical practice [7]. Therefore, a wide variety of instruments have been used to assess changes in these outcomes in the NSCLC literature.

When selecting the most appropriate outcome measure the clinician or researcher should consider the measurement properties established for their population of interest. Reliability determines the ability of an instrument to obtain data which are accurate, consistent and have small measurement errors when the instrument is repeated longitudinally (intra-rater reliability) or by multiple examiners (inter-rater reliability) $[8,9]$. Validity determines the ability of an instrument to measure what it is intended to measure, that is, how well the data relate to data obtained from the gold standard instrument (criterion-concurrent validity); how well data predict an outcome (criterion-predictive validity); or how well an instrument obtains data, as hypothesised, when compared to an instrument measuring a similar construct (construct validity) [8,9]. Responsiveness determines the ability of an instrument to detect meaningful change over time [9]. Whilst a test may have excellent reliability, validity and responsiveness in one clinical population, these findings cannot always be extrapolated to other populations [9].

This review is designed to capture outcome measures applicable for use in the clinical setting by health professionals or researchers. The COnsensus-based Standards for the selection of health status Measurement INstruments (COSMIN) guidelines and the Preferred Reporting Items for Systematic Reviews and Meta-analyses (PRISMA) guidelines have been followed to report this review $[8,10,11]$.

\section{Objectives}

1. To identify non-laboratory outcome measures which have been used to assess functional capacity, physical activity or muscle strength in participants with NSCLC;

2. To evaluate, synthesise and compare the measurement properties established in participants with NSCLC for each of the outcome measures identified.

\section{Method}

\section{Protocol}

No protocol had been previously published for this review.

The search for this systematic review was conducted in two parts. Search 1 identified studies which used an outcome measure to assess functional capacity, physical activity or muscle strength in participants with NSCLC. This initial search allowed a list of outcome measures to be generated. Search 2 identified studies which examined the measurement properties of the outcome measures identified in Search 1, specifically in participants with NSCLC.

\section{Search 1: outcome measures Eligibility criteria}

Studies This review considered any type of quantitative study design as defined by the National Health and Medical Research Council Classification [12]. Full manuscripts published in English in a peer reviewed journal from 1980 onwards were eligible.

Participants Participants of any age, diagnosed with NSCLC, at any stage of the disease were considered. NSCLC was defined as: carcinoma of the lung including adenocarcinoma, squamous cell carcinoma and large cell carcinoma [13]. At least five participants with NSCLC were required for the study to be included. Studies which included mixed cancer cohorts were also eligible providing at least five participants were diagnosed with NSCLC. The authors were contacted for studies which did not specify the type of lung cancer to confirm the number of participants with NSCLC. Studies without original participant data (such as reviews, narratives or editorials) were excluded.

Outcomes Outcomes of interest were objective tests which, based on face validity, aimed to measure functional capacity, physical activity or muscle strength in the clinical setting. Outcome measures conducted in a laboratory were excluded. Patient-reported outcome measures, such as questionnaires, were excluded.

\section{Information sources, search and study selection}

Prior to conducting this review the Cochrane Library (including the Cochrane Database of Systematic Reviews and Database of Abstract of Review of Effectiveness DARE), Physiotherapy Evidence Database (PEDro), the 
COSMIN list of systematic reviews of measurement properties [14] and the International Prospective Register of Systematic Reviews (PROSPERO) [15] were searched to ensure no similar reviews had been published. Seven electronic databases were searched by one reviewer (CG) using a systematic, comprehensive and reproducible search strategy to identify all published studies (Additional file 1). Databases were accessed via The University of Melbourne and Austin Health, Australia, with the last search run on 4-October-2012.

Search terms used were: lung cancer, NSCLC, fitness, exercise, exercise capacity, functional capacity, function, acceleromet*, physical activity monitor*, global positioning system, strength, walk*, ambulat*, pedometer*, gait, outcome, assessment, test*, functional assessment, outcome assessment, exercise test, treatment outcome, data collection. A standardised eligibility assessment was performed by two independent reviewers (CG, SP) (Additional file 1). All studies identified by the search strategy were assessed based on title/abstract for eligibility. If there was insufficient information to include/exclude a study, full-text was retrieved. Consensus was required by both reviewers. Full-text of all relevant studies was obtained and read to ensure the inclusion criteria were met. Disagreements were settled by a third independent reviewer (LD). If there was insufficient information to include/exclude an article, the authors were contacted where possible. At each assessment stage agreement between reviewers was estimated with percentage agreement and the Kappa statistic using SPSS for Windows statistical software package $\left(\right.$ IBM $^{\circledR}$ SPSS $^{\circledR}$ Statistics Version20.0.0) [16]. All references were stored in Endnote software 2010 versionX4.

\section{Data collection process}

A data collection form was specifically developed and used to extract data from studies by one reviewer (CG) and a second reviewer cross-checked extracted data (SP). To avoid double counting data, multiple reports on the same patient group were identified by juxtaposing study details. Collected data were stored in Microsoft ${ }^{(\mathrm{R})}$ Office Excel $^{(\mathrm{R})} 2007$.

\section{Search 2: measurement properties Eligibility criteria}

Studies Studies which aimed to develop an outcome measure or evaluate the measurement properties of an outcome measure identified in Search 1 were eligible. Only studies published in a peer reviewed journal were included. Conference abstracts or studies not published in a peer reviewed journal were excluded due to the inability to effectively evaluate risk of bias of the individual study. Only studies published from 1 January 1980 that were available in English were eligible.
Participants Participants of any age, diagnosed with NSCLC, at any stage of the disease were considered. NSCLC was defined as: carcinoma of the lung including adenocarcinoma, squamous cell carcinoma and large cell carcinoma [13]. At least five participants with NSCLC were required for the study to be included. Studies which included mixed cancer cohorts were also eligible providing at least five participants were diagnosed with NSCLC. The authors were contacted for studies which did not specify the type of lung cancer to confirm the number of participants with NSCLC. Studies without original participant data (such as reviews, narratives or editorials) were excluded.

Outcomes Outcomes of interest were the measurement properties: reliability (inter- or intra-rater), measurement error, criterion validity (concurrent or predictive), construct validity (hypothesis testing) and responsiveness of outcome measures identified in Search 1 [8]. Studies validating an alternative test against an outcome measure of interest (which provide indirect evidence for validity) and longitudinal studies (which provide indirect evidence for responsiveness) were excluded because such studies have not specifically formulated or tested hypotheses about the measurement properties [8]. Studies evaluating a battery measure including a relevant sub-component were also excluded as they are designed to be used in their entirety.

\section{Information sources, search and data extraction}

Four electronic databases were searched by one reviewer (CG) using a systematic, comprehensive and reproducible search strategy (Figure 1). The last search was run on 4October-2012. A previously published search filter was used (sensitivity 97.4\%; precision 4.4\%) (Additional file 2) [17]. No publication date or language restrictions were imposed on the search. The study selection and data collection processes followed were the same as described for Search 1. Data items extracted were adapted from the COSMIN generalizability checklist [10].

\section{Risk of bias of studies}

Two independent reviewers (CG, CO) evaluated risk of bias using the 4-point COSMIN checklist [18]. This checklist was originally developed to assess the methodological quality of patient-reported outcome measures however it has also been suggested for use to assess the quality of non-patient reported outcome measures [10]. Four items from the checklist (internal consistency, structural validity, cross-cultural validity and content validity) are only applicable to questionnaires and were therefore not assessed [19]. Questions for remaining items (reliability, measurement error, hypothesis testing, criterion validity and responsiveness) were scored on a 4-point scale. The overall score for each item was obtained by using the lowest 


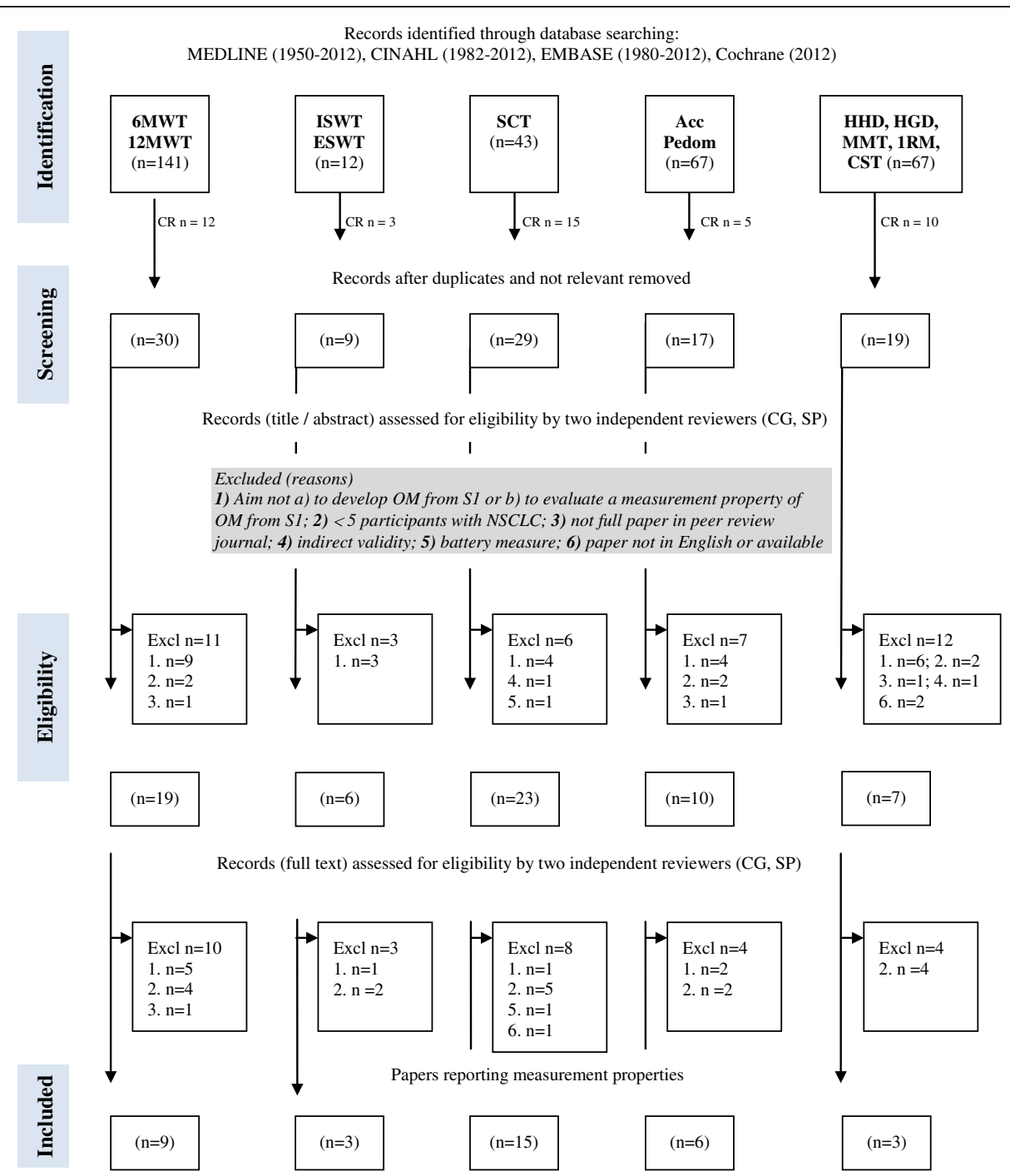

Figure 1 Flow diagram of measurement properties study selection process - Search 2. Abbreviations: 1RM, one repetition maximum; $6 \mathrm{MWT}$, six-minute walk test; 12MWT, twelve-minute walk test; Acc, accelerometer; CINAHL, Cumulative Index to Nursing and Allied Health Literature; CR, cross referencing; CST, chair-stand test; ESWT, endurance-shuttle walk test; EMBASE, the Excerpta Medica Database; excl, excluded; HHD, hand-held dynamometry; HGD, hand-grip dynamometry; ISWT, incremental-shuttle walk test; MMT, manual muscle test; n, number; NSCLC, non-small cell lung cancer; OM, outcome measure; Pedom, pedometer; S1, search from part one; SCT, stair-climb test.

score (excellent, good, fair or poor) recorded for any question within the item, as recommended by the COSMIN scoring system [18]. Reviewer agreement was estimated with percentage agreement and the Kappa statistic [16].

\section{Results}

\section{Search 1: outcome measures}

The search of seven electronic databases and cross referencing identified 6,398 studies. Assessment of title/abstract and full text results in 88 articles using 13 different outcome measures being included (Figure 1; Additional file 1). A list of outcome measures was generated (Table 1). Almost perfect agreement between reviewers of potentially relevant titles/abstracts (CG, SP)
(97.0\%, Kappa=0.93) and full-text articles (CG, SP) (94.5\%, Kappa $=0.82$ ) was obtained [16]. The third reviewer (LD) was consulted twice. Twenty-two authors were contacted to clarify the cancer type, 13 responded. In ten cases the lung cancer type could not be confirmed and these studies were excluded.

\section{Search 2: measurement properties}

Study selection

The search identified 375 studies of which 34 articles (31 studies) were included (Figure 1). Almost perfect agreement was obtained between reviewers (CG, SP) for titles/abstracts $(96 \%$, Kappa $=0.92)$ and substantial agreement was obtained for full-text articles $(90 \%$, Kappa $=0.78)$ 
Table 1 Synthesis of evidence regarding measurement properties: comparison of outcome measures

\begin{tabular}{|c|c|c|c|c|}
\hline Outcome measure & $\begin{array}{l}\text { Reliability, measurement } \\
\text { error and responsiveness }\end{array}$ & $\begin{array}{l}\text { Criterion concurrent } \\
\text { validity (gold standard) }\end{array}$ & Criterion predictive validity & $\begin{array}{l}\text { Construct validity } \\
\text { (hypothesis testing) }\end{array}$ \\
\hline \multicolumn{5}{|l|}{ Functional capacity } \\
\hline 6-minute walk test & $x$ & $x$ & $\begin{array}{l}8 \text { studies [22-27,39-41], predicts: } \\
\text { survival, post-op outcomes } \\
\text { (complications, LOS, 6MWT, } \\
\text { HRQoL) and development of RP }\end{array}$ & $\begin{array}{l}1 \text { study }[26,27] \text {, strongly } \\
\text { correlated with RFT }\end{array}$ \\
\hline 12-minute walk test & $x$ & $x$ & $x$ & $x$ \\
\hline Incremental-shuttle walk test & $x$ & $\begin{array}{l}1 \text { study [30], strongly } \\
\text { correlated with CPET }\end{array}$ & $\begin{array}{l}2 \text { studies [30,64], predicts: } \\
\text { survival and post-op outcomes }\end{array}$ & $\begin{array}{l}3 \text { studies [30,44,64], moderately } \\
\text { correlated with muscle strength; } \\
\text { little, moderate and strong } \\
\text { correlation with RFT; little } \\
\text { correlation with mastery } \\
\text { questionnaire }\end{array}$ \\
\hline Endurance-shuttle walk test & $x$ & $x$ & $x$ & $x$ \\
\hline Stair-climb test & $x$ & $\begin{array}{l}1 \text { study [29], strongly } \\
\text { correlated with CPET }\end{array}$ & $\begin{array}{l}13 \text { studies [21,22,32-39,41-43], } \\
\text { predicts: post-op outcomes } \\
\text { (complications, LOS, cost) } \\
\text { and survival }\end{array}$ & $\begin{array}{l}2 \text { studies [20,31], strong } \\
\text { correlation between } \mathrm{VO}_{2} \text { peak } \\
\text { and altitude reached; direct } \\
\text { association between pre and } \\
\text { post-op SCT results }\end{array}$ \\
\hline \multicolumn{5}{|l|}{ Physical activity } \\
\hline Accelerometer & $x$ & $x$ & $x$ & $\begin{array}{l}3 \text { studies [46-51], strongly } \\
\text { correlated with estimated EE } \\
\text { and sleep; moderately correlated } \\
\text { with depression, HRQoL and PS }\end{array}$ \\
\hline Pedometer & $x$ & $x$ & $x$ & $\begin{array}{l}1 \text { study [53], moderately } \\
\text { correlated with CPET }\end{array}$ \\
\hline \multicolumn{5}{|l|}{ Muscle strength } \\
\hline Hand-held dynamometry & $x$ & $x$ & $x$ & $x$ \\
\hline $\begin{array}{l}\text { Hand-held dynamometry } \\
\text { with pulley force sensor }\end{array}$ & $\begin{array}{l}1 \text { study [52], very } \\
\text { good intra-rater } \\
\text { reliability; large SEM }\end{array}$ & $x$ & $x$ & $x$ \\
\hline Hand-grip dynamometry & $\begin{array}{l}1 \text { study [28], moderate } \\
\text { intra-rater reliability }\end{array}$ & $x$ & $x$ & $x$ \\
\hline Manual-muscle test & $x$ & $x$ & $x$ & $x$ \\
\hline One-repetition maximum & $x$ & $x$ & $x$ & $x$ \\
\hline Chair-stand test & $x$ & $x$ & $x$ & $\begin{array}{l}1 \text { study [53], moderately } \\
\text { correlated with PS and fatigue }\end{array}$ \\
\hline
\end{tabular}

Abbreviations: $6 M W T$, six-minute walk test; $C P E T$, cardio-pulmonary exercise testing; $E E$, energy expenditure; $H R Q o L$, health related quality of life; ICC, intraclass correlation coefficient; $L O S$, length of hospital stay; post-op, post-operative; pre-op, pre-operative; $P S$, Performance Status; RFT, respiratory function tests; $R P$, radiation pneumonitis; $S C T$, stair-climb test; $S E M$, standard error of measurement; $V_{2}$ peak, peak oxygen consumption.

$\mathrm{x}=$ not assessed.

[16]. Twelve authors were contacted to clarify the cancer type, nine responded. In seven cases the lung cancer type could not be confirmed and these studies were excluded.

\section{Study characteristics}

Table 2 summarises the 31 prospective observational studies. The majority of studies included only participants with NSCLC $(\mathrm{n}=18,58 \%)$. Studies had a mean (standard deviation [SD]) sample size of 130 (146) participants (range 12-640). Outcome measures were longitudinally repeated in $25 \%$ of studies: before and after surgery $(\mathrm{n}=5,16 \%)$ [20-24], chemotherapy $(\mathrm{n}=1,3 \%)$ [25] and radiotherapy $(\mathrm{n}=2,6 \%)$ [26-28] (Table 3$)$.

\section{Outcome measures}

Measurement properties evaluated were: intra-rater reliability (studies $\mathrm{n}=1$ ); inter-rater reliability $(\mathrm{n}=1)$; measurement error $(\mathrm{n}=1)$; criterion-concurrent validity $(\mathrm{n}=2)$; criterion-predictive validity $(\mathrm{n}=20)$; construct validity (hypothesis testing) $(\mathrm{n}=11)$ and responsiveness $(\mathrm{n}=0)$ (Table 1; Table 4; Additional file 3).

\section{Risk of bias of studies}

Risk of bias was assessed by independent reviews (CG, CO) achieving a percentage agreement of $87 \%$, Kappa $=0.80$ [16]. Consensus was achieved on $100 \%$ of occasions that reviewers disagreed. Overall studies evaluating validity scored 
Table 2 Study characteristics - part 2

\begin{tabular}{|c|c|c|c|c|c|c|c|c|}
\hline Author, yr location & n & $\begin{array}{l}\text { Gender } \\
\mathrm{M} / \mathrm{F}\end{array}$ & $\begin{array}{l}\text { Age mean } \\
\text { (SD) yrs }\end{array}$ & OM & $\begin{array}{l}\text { Setting }(n) \% \text { / method } \\
\text { of pt selection }\end{array}$ & Cancer type $(\mathbf{n}) \%$ & Cancer stage (n)\% & $\begin{array}{l}\text { Cancer treatment at } \\
\text { baseline time-point }(\mathrm{n}) \%\end{array}$ \\
\hline \multicolumn{9}{|l|}{ Functional capacity } \\
\hline Jones 2012 USA [40] & 118 & $71 / 47$ & $61 \pm 10$ & $6 \mathrm{MWT}$ & $\begin{array}{l}\text { Health system / } \\
\text { consecutive }\end{array}$ & NSCLC & IIIB, IV, recurrent IV & $\begin{array}{l}\text { Chemo (70) 59\%; RT (10) 8\%; } \\
\text { post-op (27) 23\%; post-chemo } \\
\text { (55) 47\%; post-RT (54) 46\% }\end{array}$ \\
\hline Pancieri 2010 Brazil [22] & 40 & $22 / 18$ & $48 \pm 16$ & $\begin{array}{l}6 \mathrm{MWT} ; \\
\mathrm{SCT}\end{array}$ & Hospital / consecutive & $\begin{array}{l}\text { NSCLC (33) 82.5\%; other LC } \\
\text { (2) } 5 \% \text {; benign (5) } 12.5 \%\end{array}$ & NR & Pre-op LR \\
\hline Kasymjanova 2009 Canada [25] & 64 & $29 / 35$ & $62.0 \pm 10.8$ & $6 \mathrm{MWT}$ & Outpt clinic / consecutive & $\mathrm{NSCLC}$ & III (8) 12\%; IV (56) 87\% & Pre-chemo \\
\hline Mao 2007* Miller 2005 USA [26,27] & 53 & $28 / 25$ & 64 (range 45-81) & $6 \mathrm{MWT}$ & $\begin{array}{l}\text { Hospital/ sample part of } \\
\text { larger prospective trial }\end{array}$ & $\begin{array}{l}\text { NSCLC (39) } 74 \% \text {; SCLC (12) 23\%; } \\
\text { meso (1) } 2 \% \text {; lung met (1) } 2 \%\end{array}$ & $\begin{array}{l}\text { NSCLC only: I - II (4) } \\
8 \% ; \text {; II-IV (41) 77\%; } \\
\text { recurrent (8) 15\% }\end{array}$ & $\begin{array}{l}\text { Pre- RT (17) 32\%; pre-chemo-RT } \\
\text { (36) 68\%; prior chemo (33) 62\%; }\end{array}$ \\
\hline Saad 2007 Brazil [24] & 36 & $20 / 16$ & Median $55.5 \pm 13.4$ & 6MWT & Hospital / consecutive & $\begin{array}{l}\text { NSCLC (26) 72\%; lung met (8) } \\
22 \% \text {; sarcoma (2) 6\% }\end{array}$ & NR & Pre-op LR \\
\hline Parsons 2003 Canada [39] & 70 & $40 / 30$ & 65 (range 29-83) & $\begin{array}{l}6 \mathrm{MWT} \\
\mathrm{SCT}\end{array}$ & Hospital / convenience & $\begin{array}{l}\text { NSCLC (55) 79\%; pulmonary } \\
\text { met (11) 16\%; meso (2) 3\%; } \\
\text { benign (2) 3\% }\end{array}$ & NR & Pre-op LR \\
\hline Pierce 1994 Australia [23] & 54 & $54 / 0$ & $67 \pm 7$ & $6 \mathrm{MWT}$ & Hospital / consecutive & LC (including NSCLC) & NR & Pre-op LR \\
\hline Holden 1992 USA [41] & 16 & $13 / 3$ & $68 \pm 9.3$ & $\begin{array}{l}\text { 6MWT; } \\
\text { SCT }\end{array}$ & Hospital & NSCLC (15) 94\%; SCLC (1) 6\% & $\begin{array}{l}\text { I (10) 62\%; II (3) 19\%; III } \\
\text { (2) } 12 \% ; \text { IV (1) } 6 \%\end{array}$ & Pre-op LR \\
\hline England 2012 UK [44] & 41 & $21 / 20$ & $64 \pm 8$ & ISWT & Outpt clinic & $\begin{array}{l}\text { NSCLC (26) 63\%; meso (11) } \\
27 \% ; \text { SCLC (4) 10\% }\end{array}$ & $\begin{array}{l}\text { Local (21) 51\%; } \\
\text { advanced (20); } 49 \%\end{array}$ & $\begin{array}{l}\text { Post pall-chemo (26) 63\%; post } \\
\text { pall-RT (10) 24\%; post-RT (1) 2\% }\end{array}$ \\
\hline Win 2006 UK [30] & 125 & $81 / 44$ & $68.8 \pm 7.7$ & ISWT & Consecutive & NSCLC & NR & Pre-op LR \\
\hline Win 2004 UK [64] & 111 & $71 / 40$ & 69 (range 42-85) & ISWT & Outpt clinic & $\begin{array}{l}\text { NSCLC (107) 96\%; miscellaneous } \\
\text { (4) } 4 \%\end{array}$ & $|-||| A$ & Pre-op LR \\
\hline Brunelli 2012 Italy [43] & 282 & $218 / 64$ & $68.0 \pm 9.8$ & SCT & $\begin{array}{l}\text { Tertiary referral centre / } \\
\text { consecutive }\end{array}$ & NSCLC & $\begin{array}{l}\text { I (118) } 42 \% \text {; other } \\
\text { (164) } 58 \%\end{array}$ & Pre-op LR \\
\hline Brunelli 2010 Italy [31] & 109 & $83 / 26$ & $66.6 \pm 11.1$ & SCT & $\begin{array}{l}\text { Tertiary referral centre / } \\
\text { consecutive }\end{array}$ & $\mathrm{NSCLC}$ & NR & Pre-op LR \\
\hline Brunelli 2008a Italy [34] & 536 & $426 / 110$ & $67 \pm 9$ & SCT & $\begin{array}{l}\text { Tertiary referral centre / } \\
\text { consecutive }\end{array}$ & NSCLC & $\begin{array}{l}\text { I (206) } 38 \% ;>1 \\
\text { (330) } 62 \%\end{array}$ & $\begin{array}{l}\text { Pre-op LR (536) 100\%; chemo } \\
\text { (56) } 10 \%\end{array}$ \\
\hline Brunelli 2008b Italy [33] & 640 & NR & $66.7 \pm 9.3$ & SCT & $\begin{array}{l}\text { Tertiary referral centre / } \\
\text { consecutive }\end{array}$ & NSCLC & NR & $\begin{array}{l}\text { Pre-op LR (640) 100\%; } \\
\text { neoadjuvant chemo (100) 16\% }\end{array}$ \\
\hline $\begin{array}{l}\text { Koegelenberg } 2008 \text { South } \\
\text { Africa [29] }\end{array}$ & 44 & $31 / 13$ & $47.6 \pm 12.5$ & SCT & $\begin{array}{l}\text { Tertiary referral centre / } \\
\text { consecutive }\end{array}$ & NSCLC (13) 29\%; benign (31) 70\% & NR & Pre-op LR \\
\hline Nikolic 2008 Croatia [36] & 101 & $82 / 19$ & $61.1 \pm 8.4$ & SCT & Hospital / consecutive & NSCLC & NR & Pre-op LR \\
\hline Brunelli 2007 Italy [20] & 200 & NR & $66.8 \pm 9.1$ & SCT & $\begin{array}{l}\text { Tertiary referral centre / } \\
\text { consecutive }\end{array}$ & NSCLC & NR & $\begin{array}{l}\text { Pre-op LR (200) 100\%; } \\
\text { neoadjuvant chemo (19) 9.5\% }\end{array}$ \\
\hline Toker 2007 Turkey [37] & 150 & $127 / 23$ & $\begin{array}{l}59.3 \pm 10.3(\mathrm{gp} \mathrm{1}) \\
60.7 \pm 10.9(\mathrm{gp} \mathrm{2})\end{array}$ & $\mathrm{SCT}$ & $\begin{array}{l}\text { University hospital / } \\
\text { consecutive }\end{array}$ & NSCLC & $N R$ & Pre-op LR \\
\hline
\end{tabular}


Table 2 Study characteristics - part 2 (Continued)

\begin{tabular}{|c|c|c|c|c|c|c|c|c|}
\hline Brunelli 2005 Italy [42] & 391 & $309 / 82$ & $\begin{array}{l}69.1 \pm 8.3(\mathrm{gp} \mathrm{1}) \\
67.0 \pm 9.0(\mathrm{gp} \mathrm{2})\end{array}$ & SCT & $\begin{array}{l}\text { Tertiary referral centre / } \\
\text { consecutive }\end{array}$ & NSCLC & NR & Pre-op LR \\
\hline Brunelli 2004 Italy [35] & 109 & NR & $75.2 \pm 3.0$ & SCT & $\begin{array}{l}\text { Tertiary referral centre / } \\
\text { consecutive }\end{array}$ & NSCLC & I (23) $21 \%$; > I (86) 79\% & Pre-op lobectomy \\
\hline Brunelli 2003 Italy [21] & 227 & NR & $\begin{array}{l}66.4 \pm 9.1(\text { gp 1) } \\
66.8 \pm 8.1(\mathrm{gp} \mathrm{2})\end{array}$ & SCT & $\begin{array}{l}\text { Tertiary referral centre / } \\
\text { consecutive }\end{array}$ & NSCLC & NR & Pre-op LR \\
\hline Brunelli 2002 Italy [32] & 160 & $128 / 32$ & $66.2 \pm 9.6$ & SCT & $\begin{array}{l}\text { Tertiary referral centre / } \\
\text { consecutive }\end{array}$ & NSCLC & NR & Pre-op LR \\
\hline Pate 1996 USA [38] & 12 & $10 / 2$ & $63.6 \pm 4.9$ & SCT & $\begin{array}{l}\text { Three university } \\
\text { hospitals / consecutive }\end{array}$ & NSCLC (7) 58\%; NR (5) 42\% & $\begin{array}{l}\text { I (5) 42\%; III (2) 17\%; } \\
\text { NR (5) } 42 \%\end{array}$ & Pre-op LR \\
\hline \multicolumn{9}{|l|}{ Physical activity } \\
\hline Maddocks 2012 UK [47] & 84 & $54 / 30$ & $66 \pm 9$ & Acc & Outpt clinic & $\begin{array}{l}\text { NSCLC (71) 84\%; SCLC (8) 9\%; } \\
\text { meso (5) 6\% }\end{array}$ & IIIB (43) 51\%; IV (41) 49\% & Palliative Rx \\
\hline $\begin{array}{l}\text { Grutsch } 2011 \mathrm{a}, 2011 \mathrm{~b} ; \\
\text { Du-Quiton } 2010 \text { USA [48-50] }\end{array}$ & 84 & $65 / 19$ & 62 (range 40-94) & Acc & $\begin{array}{l}\text { Hospital inpt (42) 50\%; } \\
\text { home (42) } 50 \%\end{array}$ & NSCLC & $\begin{array}{l}\text { II (1) 1\%; III (18) 21\%; } \\
\text { IV (65) 77\% }\end{array}$ & $\begin{array}{l}\text { Pre-chemo (84) 100\%; prior Rx } \\
\text { (31) } 37 \%\end{array}$ \\
\hline Maddocks 2010 UK [46] & 60 & $40 / 20$ & $68 \pm 9$ & Acc & Outpt clinic & $\begin{array}{l}\text { NSCLC (53) 88\%: meso (5) } \\
8 \% ; \text { Gl (2) 3\% }\end{array}$ & $\begin{array}{l}\text { Local (35) 58\%; } \\
\text { advanced (25) } 42 \%\end{array}$ & NR \\
\hline Novoa 2011 Spain [51] & 38 & $30 / 8$ & $62.8 \pm 10.1$ & Pedom & $\begin{array}{l}\text { Output clinic / } \\
\text { consecutive }\end{array}$ & NSCLC & NR & Pre-op LR \\
\hline \multicolumn{9}{|l|}{ Muscle strength } \\
\hline Trutschnigg 2008 Canada [28] & 74 & $48 / 26$ & $61.5 \pm 13.1$ & HGD & Hospital and laboratory & NSCLC; Gl & Advanced & NR \\
\hline Brown 2005 UK [53] & 53 & $30 / 23$ & $\begin{array}{l}\text { Median } 64 \\
\text { (range 43-81) }\end{array}$ & HGD CST & $\begin{array}{l}\text { Palliative care centre } \times 2 \\
\text { and hospital }\end{array}$ & $\begin{array}{l}\text { Gp1: healthy controls (15) 100\% } \\
\text { Gp2: cancer: NSCLC (29) 76\%; SCLC } \\
\text { (6) 16\%; no histology LC (3) 8\% }\end{array}$ & $\begin{array}{l}\text { Locally advanced (20) } \\
53 \% ; \text { IV (18) } 47 \%\end{array}$ & NR \\
\hline Knols 2002 Switzerland [52] & 40 & $27 / 13$ & $49.4 \pm 14.8$ & $\begin{array}{l}\mathrm{HHD+} \\
\text { pulley }\end{array}$ & $\begin{array}{l}\text { Hospital inpt / } \\
\text { convenience }\end{array}$ & $\begin{array}{l}\text { NSCLC (7) 17.5\%; other LC (3) 7.5\%; } \\
\text { haem (20) 50\%; sarcoma (5) 12.5\%; } \\
\text { seminoma (3) 7.5\%; other (2) 5\% }\end{array}$ & I-IV & Chemo \\
\hline
\end{tabular}

Abbreviations: $6 M W T$, six-minute walk test; $A c c$, accelerometer; ca, cancer; chemo, chemotherapy; $C S T$, chair-stand test; $F$, female; gp, group; gyn, gynaecological; haem, haematological; $G l$, gastro-intestinal; $H H D$, handheld dynamometry; $H G D$, hand-grip dynamometry; $H T$, hormone therapy; ISWT, incremental shuttle walk test; inpt, inpatient; $L C$, lung cancer; $L R$, lung resection; $M$, male; meso, mesothelioma; met, metastasis; $n$, number of participants; NR, not reported; NSCLC, non-small cell lung cancer; OM, outcome measure; outpt; outpatient; pall, palliative; pedom, pedometer; PFS, pulley-force sensor; post-op, post-operative; pre-op, pre-operative; $p t$, participant; $R T$, radiotherapy; $R x$, treatment; stair, stair-climbing test; SCLC, small cell lung cancer; SCT, stair-climb test; SD, standard deviation; wks, weeks; yr, year published; yrs, years.

* data presented from most recent publication. 
Table 3 Description of outcome measures used

\begin{tabular}{|c|c|c|c|c|c|c|c|}
\hline \multicolumn{8}{|l|}{ Author, yr } \\
\hline $6 M W T$ & \multicolumn{2}{|l|}{$\begin{array}{l}\text { OM procedure } \\
\text { referenced }\end{array}$} & \multicolumn{2}{|l|}{ Encouragement standardised } & \multicolumn{3}{|l|}{$\begin{array}{l}\text { Number of repeat } \\
\text { Ax in testing session }\end{array}$} \\
\hline Jones 2012 [40] & Yes [50] & & NR & & NR & & \\
\hline Pancieri 2010 [22] & No & & 'encouraged walking' & & NR & & \\
\hline $\begin{array}{l}\text { Kasymjanova } \\
2009[25]\end{array}$ & Yes $[50,67]$ & & NR & & $\begin{array}{l}1 \times \text { practice } 1 \times \text { actual } \\
\text { (on different days) }\end{array}$ & & \\
\hline $\begin{array}{l}\text { Mao 2007, Miller } \\
2005[26,27]\end{array}$ & Yes [20] & & NR & & NR & & \\
\hline Saad 2007 [24] & Yes [50] & & $N R$ & & NR & & \\
\hline Parsons 2003 [39] & Yes [34,68-72] & & Yes & & $1 \times$ practice, $1 \times$ actual & & \\
\hline Pierce 1994 [23] & Yes [73] & & $N R$ & & 3 (best value used) & & \\
\hline Holden 1992 [41] & No & & $N R$ & & 2 (15-30 min interval) & & \\
\hline ISWT & $\begin{array}{l}\text { OM procedure } \\
\text { referenced }\end{array}$ & & Encouragement standardised & & $\begin{array}{l}\text { No of repeat } A x \text { in } \\
\text { testing session }\end{array}$ & & Additional description \\
\hline England 2012 [44] & Yes [59] & & $N R$ & & $\begin{array}{l}1 \times \text { practice, } \\
1 \times \text { actual }\end{array}$ & & $\begin{array}{l}\text { Participants wore COSMED } \mathrm{K} 4 \mathrm{~b}^{2} \\
\text { system (COSMED, Italy) to measure } \\
\mathrm{HR}, \mathrm{VE} \text { and } \mathrm{VO}_{2} \text { throughout test }\end{array}$ \\
\hline $\begin{array}{l}\text { Win 2006; } 2004 \\
{[30,64]}\end{array}$ & Yes [59] & & $N R$ & & 1 & & $\begin{array}{l}\mathrm{HR} \text { and } \mathrm{SpO}_{2} \text { monitored and recorded } \\
\text { at } 30 \text { second intervals throughout test }\end{array}$ \\
\hline$S C T$ & Equipment & $\begin{array}{l}\text { Monitoring } \\
\text { during test }\end{array}$ & Intensity & Outcome & $\begin{array}{l}\text { Number of repeat } \\
\text { Ax in testing session }\end{array}$ & $\begin{array}{l}\text { Experience } \\
\text { of assessors }\end{array}$ & Additional description \\
\hline \multirow[t]{4}{*}{$\begin{array}{l}\text { Brunelli 2012, 2010, } \\
\text { 2008a, 2008b, 2007, } \\
\text { 2005, 2004, 2003, } \\
\text { 2002 [20-21, 31, } \\
\text { 32-35, 42-43,] }\end{array}$} & $\begin{array}{l}16 \text { flights } \times 11 \text { steps } \\
(0.155 \mathrm{~m} \text { height })\end{array}$ & $\begin{array}{l}\text { Continuous verbal } \\
\text { interaction to } \mathrm{Ax} \\
\mathrm{SOB} / \text { symptoms; } \\
\mathrm{HR} ; \mathrm{SpO}_{2}\end{array}$ & $\begin{array}{l}\text { Pace of pt choice, asked to } \\
\text { climb max no steps and stop } \\
\text { for exhaustion, limiting SOB, } \\
\text { leg fatigue or chest pain }\end{array}$ & $\begin{array}{l}\text { No steps and time } \\
\text { taken, minimum value } \\
\mathrm{SpO}_{2} \text {, exercise oxygen } \\
\text { desaturation (= fall } \\
\mathrm{SpO}_{2}<90 \% \text { or } \\
\text { fall }>4 \% \text { ) }\end{array}$ & 1 & Physician & Calculations from test: \\
\hline & & & & & & & $\begin{array}{l}\text { - Work }=(\text { step height in meters } x \\
\text { steps per min } \times \text { body weight in } \\
\mathrm{kg} \times 0.1635)[74]\end{array}$ \\
\hline & & & & & & & $\begin{array}{l}\cdot \mathrm{VO}_{2} \max (\mathrm{ml} / \mathrm{min})=5.8 \times \text { weight } \\
\text { in } \mathrm{kg}+151+10.1 \times \text { work) }[74]\end{array}$ \\
\hline & & & & & & & $\begin{array}{l}-\mathrm{VO}_{2} \max \text { corrected for body surface } \\
\text { area }\left(\mathrm{ml} / \mathrm{min}^{2}\right)=\mathrm{VO}_{2} \max / \max \mathrm{HR}\end{array}$ \\
\hline Pancieri 2010 [22] & $\begin{array}{l}6 \text { flights } \times 12 \text { steps } \\
(16.9 \mathrm{~cm} \text { height), } \\
30^{\circ} \text { incline }\end{array}$ & $\mathrm{HR}, \mathrm{SpO}_{2}$ & $\begin{array}{l}\text { Climb all steps in the shortest } \\
\text { possible time with verbal } \\
\text { encouragement between flights. } \\
\text { Testing stopped for fatigue, } \\
\text { limiting SOB, thoracic pain or } \\
\text { exhaustion }\end{array}$ & Time taken & 1 & & \\
\hline
\end{tabular}


Table 3 Description of outcome measures used (Continued)

\begin{tabular}{|c|c|c|c|c|c|c|c|}
\hline $\begin{array}{l}\text { Koegelenberg } \\
2008[29]\end{array}$ & $\begin{array}{l}12 \text { flights } \times 10 \text { steps } \\
(3.48 \mathrm{~m} \mathrm{~b} / \mathrm{t} \text { floors })\end{array}$ & $\mathrm{HR}, \mathrm{SpO}_{2}$ & $\begin{array}{l}\text { Pt asked to climb 'as fast and as } \\
\text { high' as they possibly could to a } \\
\text { max elevation of } 20 \mathrm{~m}\end{array}$ & $\begin{array}{l}\text { Altitude, time taken, } \\
\text { speed of ascent }\end{array}$ & NR & NR & $\begin{array}{l}\text { Stair climb considered completed } \\
\text { if pt rested or more than } 3 \text { seconds } \\
\text { or reached } 20 \mathrm{~m} \text { height Allowed to } \\
\text { use rail only for balance }\end{array}$ \\
\hline Nikolic 2008 [36] & $\begin{array}{l}92 \text { steps } \\
\text { ( } 0.15 \mathrm{~m} \text { height) }\end{array}$ & $\begin{array}{l}\mathrm{HR}, \mathrm{SpO}_{2} \\
\text { (measured every } \\
20 \text { steps) }\end{array}$ & $\begin{array}{l}\text { Pace of pt choice, asked to climb } \\
\text { max no steps and stop for } \\
\text { exhaustion, limiting SOB, leg } \\
\text { fatigue or chest pain }\end{array}$ & $\begin{array}{l}\text { No steps, time } \\
\text { taken }\end{array}$ & 1 & Physician & Pt instructed not to use hand-rail \\
\hline Toker 2007 [37] & $\begin{array}{l}20 \text { steps per flight } \\
(15 \mathrm{~cm} \text { height) }\end{array}$ & $\mathrm{HR}, \mathrm{SpO}_{2}$ & $\begin{array}{l}\text { Pt motivated to do their best } \\
\text { and motivation }\end{array}$ & $\begin{array}{l}\mathrm{SpO}_{2} \text { (pre, post, } \\
\text { change), } \mathrm{HR} \text { (pre, } \\
\text { post, change), } \\
\text { time taken }\end{array}$ & 2 & $\begin{array}{l}\text { Resident } \\
\text { doctor }\end{array}$ & \\
\hline Pate 1996 [38] & $\begin{array}{l}21 \text { steps per flight } \\
(17.5 \mathrm{~cm} \text { height) }\end{array}$ & $\mathrm{HR}, \mathrm{SpO}_{2}$ & $\begin{array}{l}\text { Moderate pace of pts choice, } \\
\text { encouraged to exercise to a } \\
\text { symptom-limited max and } \\
\text { complete the flight of stairs they } \\
\text { were on if possible }\end{array}$ & $\begin{array}{l}\text { No steps, time } \\
\text { taken, altitude }(\mathrm{m}), \\
\text { reason for stopping }\end{array}$ & 1 & NR & $\begin{array}{l}\text { Test considered completed as soon } \\
\text { as patient stopped for any reason } \\
\text { Pt instructed not to use hand-rail }\end{array}$ \\
\hline \multirow[t]{3}{*}{ Holden 1992 [41] } & $\begin{array}{l}11 \text { steps per flight } \\
\text { (0.17 m height) }\end{array}$ & $\mathrm{SpO}_{2}$ & Own pace & Altitude, time taken & 1 & Therapist & Calculations from test: \\
\hline & & & & & & & $\begin{array}{l}\text { - Work }=\text { step height } \times \text { steps per } \\
\text { min } \times \text { weight in } \mathrm{kg} \times 0.1635\end{array}$ \\
\hline & & & & & & & $\begin{array}{l}\cdot \mathrm{VO}_{2} \mathrm{ml} / \mathrm{min}=5.8 \times \text { weight in } \\
\mathrm{kg}+151+(10.1 \times \text { work })\end{array}$ \\
\hline Physical activity & Equipment & & Location of monitoring & Outcome & Duration of $A x$ & $\begin{array}{l}\text { Position of } \\
\text { sensor }\end{array}$ & \\
\hline $\begin{array}{l}\text { Maddocks } \\
2012[47]\end{array}$ & $\begin{array}{l}\text { Uni-axial accelerometer } \\
\text { ActivPAL }{ }^{\text {TM }} \text { monitor } \\
\text { (PAL Technologies } \\
\text { Ltd., UK) }\end{array}$ & $\begin{array}{l}\text { Home } \\
\text { environment }\end{array}$ & $\begin{array}{l}\text { Mean daily step count, number } \\
\text { of sit-to-stand transitions, time in } \\
\text { hrs spent sitting/lying, upright } \\
\text { standing and upright stepping }\end{array}$ & $\begin{array}{l}7 \text { days ( } 6 \text { full } \\
\text { days of data) }\end{array}$ & $\begin{array}{l}\text { Mid-third of anterior } \\
\text { thigh of pts chosen leg }\end{array}$ & & \\
\hline $\begin{array}{l}\text { Grutsch 2011a, } \\
2011 \text { b; Du-Quiton } \\
2010 \text { [48-50] }\end{array}$ & $\begin{array}{l}\text { Actigraph Piezoelectric } \\
\text { Accelerometer } \\
\text { (Ambulatory } \\
\text { Monitoring Inc., } \\
\text { AMI, USA) }\end{array}$ & $\begin{array}{l}\text { Group } 1 \text { (inpt) } \\
\text { and group } 2 \\
\text { (home environment) }\end{array}$ & $\begin{array}{l}\text { Mean duration daytime activity } \\
\text { (no of vertical/horizontal } \\
\text { accelerations per min) }\end{array}$ & 3-7 days & Non-dominant wrist & & \\
\hline Novoa 2011 [51] & $\begin{array}{l}\text { OMROM Walking } \\
\text { Style Pro }{ }^{\circledR} \text { pedometer }\end{array}$ & Home environment & $\begin{array}{l}\text { Mean daily no total and aerobic } \\
\text { steps; mean daily distance } \\
\text { walked (km); mean daily time } \\
\text { of aerobic activity (min) }\end{array}$ & $\begin{array}{l}\text { Daytime only, daily } \\
\text { Ax while waiting } \\
\text { for surgery }\end{array}$ & $\begin{array}{l}\text { Pedometer attached } \\
\text { to waist band or belt }\end{array}$ & & \\
\hline $\begin{array}{l}\text { Maddocks } \\
2010[46]\end{array}$ & $\begin{array}{l}\text { Uni-axial accelerometer } \\
\text { ActivPAL }{ }^{\text {TM }} \text { monitor } \\
\text { (PAL Technologies } \\
\text { Ltd., UK) }\end{array}$ & Home environment & $\begin{array}{l}\text { Mean daily step count and } \\
\text { estimate energy expenditure } \\
\text { (METh); acceptability } \\
\text { (non-compliance in hours); } \\
\text { optimal duration of monitoring }\end{array}$ & $\begin{array}{l}7 \text { days ( } 6 \text { full } \\
\text { days of data) }\end{array}$ & $\begin{array}{l}\text { Mid-third of anterior } \\
\text { thigh of dominant leg }\end{array}$ & & \\
\hline
\end{tabular}


Table 3 Description of outcome measures used (Continued)

\begin{tabular}{|c|c|c|c|c|c|c|c|}
\hline Physical activity & Equipment & & Location of monitoring & Outcome & Duration of $A x$ & $\begin{array}{l}\text { Position } \\
\text { of sensor }\end{array}$ & \\
\hline $\begin{array}{l}\text { Maddocks } \\
2012[47]\end{array}$ & $\begin{array}{l}\text { Uni-axial accelerometer } \\
\text { ActivPAL }{ }^{T M} \text { monitor } \\
\text { (PAL Technologies } \\
\text { Ltd., UK) }\end{array}$ & Home environment & $\begin{array}{l}\text { Mean daily step count, number } \\
\text { of sit-to-stand transitions, time } \\
\text { in hrs spent sitting/lying, upright } \\
\text { standing and upright stepping }\end{array}$ & $\begin{array}{l}7 \text { days ( } 6 \text { full } \\
\text { days of data) }\end{array}$ & $\begin{array}{l}\text { Mid-third of anterior } \\
\text { thigh of pts chosen leg }\end{array}$ & & \\
\hline $\begin{array}{l}\text { Grutsch } 2011 \mathrm{a}, \\
2011 \mathrm{~b} \text {; Du-Quiton } \\
2010 \text { [48-50] }\end{array}$ & $\begin{array}{l}\text { Actigraph Piezoelectric } \\
\text { Accelerometer } \\
\text { (Ambulatory Monitoring } \\
\text { Inc., AMl, USA) }\end{array}$ & $\begin{array}{l}\text { Group } 1 \text { (inpt) } \\
\text { and group } 2 \\
\text { (home environment) }\end{array}$ & $\begin{array}{l}\text { Mean duration daytime activity } \\
\text { (no of vertical/horizontal } \\
\text { accelerations per min) }\end{array}$ & 3-7 days & Non-dominant wrist & & \\
\hline Novoa 2011 [51] & $\begin{array}{l}\text { OMROM Walking Style } \\
\text { Pro }{ }^{\circledR} \text { pedometer }\end{array}$ & Home environment & $\begin{array}{l}\text { Mean daily no total and aerobic } \\
\text { steps; mean daily distance } \\
\text { walked (km); mean daily time } \\
\text { of aerobic activity (min) }\end{array}$ & $\begin{array}{l}\text { Daytime only, daily } \\
\text { Ax while waiting } \\
\text { for surgery }\end{array}$ & $\begin{array}{l}\text { Pedometer attached } \\
\text { to waist band or belt }\end{array}$ & & \\
\hline $\begin{array}{l}\text { Maddocks } \\
2010[46]\end{array}$ & $\begin{array}{l}\text { Uni-axial accelerometer } \\
\text { ActivPAL }{ }^{T M} \text { monitor } \\
\text { (PAL Technologies } \\
\text { Ltd., UK) }\end{array}$ & Home environment & $\begin{array}{l}\text { Mean daily step count and } \\
\text { estimate energy expenditure } \\
\text { (METh); acceptability } \\
\text { (non-compliance in hours); } \\
\text { optimal duration of monitoring }\end{array}$ & $\begin{array}{l}7 \text { days ( } 6 \text { full } \\
\text { days of data) }\end{array}$ & $\begin{array}{l}\text { Mid-third of anterior } \\
\text { thigh of dominant leg }\end{array}$ & & \\
\hline Muscle strength & Equipment & $\begin{array}{l}\text { Muscle group } \\
\text { movement }\end{array}$ & Participant position & No of assessors & $\begin{array}{l}\text { Number of repeat } \\
\text { Ax in testing session }\end{array}$ & $\begin{array}{l}\text { Experience } \\
\text { of assessors }\end{array}$ & Additional description \\
\hline $\begin{array}{l}\text { Trutschnigg } \\
2008[28]\end{array}$ & $\begin{array}{l}\text { Jamar HGD (Sammons } \\
\text { Preston, Bolingbrook), } \\
\text { position } 3 \text { on handle }\end{array}$ & Grip & $\begin{array}{l}\text { Sitting, feet on ground shoulder } \\
\text { width apart, Elb } 90^{\circ} \mathrm{F} \text {, wrist } 0^{\circ} \text {, } \\
\text { arm on arm rest [75] }\end{array}$ & NR & $\begin{array}{l}1-2 \times \text { practice } \\
2 \times 3 \text { reps actual } \\
\text { (mean value used) }\end{array}$ & $N R$ & $\begin{array}{l}\text { Dominant hand Patient instructed } \\
\text { when to start and stop contraction } \\
\text { with a } 3 \text { second contraction time } \\
\text { No encouragement }\end{array}$ \\
\hline Brown 2005 [53] & $\begin{array}{l}\text { Square design chair } \\
\text { with firm seat } 43 \mathrm{~cm} \\
\text { height and arm rests }\end{array}$ & Sit to stand & Seated & NR & NR & NR & $\begin{array}{l}\text { Pt asked to rise from seated position } \\
\text { to fully upright position as fast as } \\
\text { they could, if possible without } \\
\text { using arm rests }\end{array}$ \\
\hline \multirow[t]{2}{*}{ Knols 2002 [52] } & \multirow[t]{2}{*}{$\begin{array}{l}\text { Mecmesin FB50K } \\
\text { pull-gauge HHD, } \\
\text { Mecmesin, England }\end{array}$} & \multirow[t]{2}{*}{ Elb E, Knee E } & $\begin{array}{l}\text { 1. Supine, Elb } 90^{\circ} \mathrm{F} \text {, upper edge } \\
\text { pull- attachment perpendicular } \\
\text { to ulnar side of forearm distal to } \\
\text { caput ulnae, non-elastic belt } \\
\text { over ASIS stabilised pt on table }\end{array}$ & \multirow[t]{2}{*}{$\begin{array}{l}2 \text { (random order) } \\
30 \text { min interval }\end{array}$} & \multirow[t]{2}{*}{$\begin{array}{l}6 \times \text { practice } 3 \times \text { actual } \\
(60 \text { second interval) }\end{array}$} & \multirow[t]{2}{*}{ Physiotherapist } & \multirow{2}{*}{$\begin{array}{l}\text { Dominant UL and LL Ax - identified } \\
\text { by asking participant to throw a ball } \\
\text { and kick a ball (preferred stance leg } \\
\text { chosen) Figures also provided for } \\
\text { both testing positions 'Make' test } \\
\text { used Pt asked to increase force } \\
\text { over } 2 \text { seconds and maintain for } \\
\text { another } 5 \text { seconds }\end{array}$} \\
\hline & & & $\begin{array}{l}\text { 2. sitting edge of table upright no } \\
\text { back support, knee } 90^{\circ} \mathrm{F} \text {, stabilise } \\
\text { trunk by grasping table, lower } \\
\text { edge pull-attachment } \\
\text { perpendicular to ant surface tibia, } \\
\text { distal to end med mall }\end{array}$ & & & & \\
\hline
\end{tabular}

Abbreviations: 1RM, one repetition maximum; 6MWT, six-minute walk test; ant, anterior; $A S I S$, spinae iliacae ant superiorum; ATS, American Thoracic Society; $A x$, assessment; bt, between; $C m$, centimeters; $d y n$, dynamometer; $E$, extension; Elb, elbow; $F$, flexion; $H H D$, hand held dynamometer; $H G D$, hand grip dynamometer; $H R$, heart rate; $h r s$, hours; ISWT, incremental shuttle walk test; $k g$, kilograms; $k m$, kilometers; $L$, left; $L L$, lower limb; $m$, meters; mal, malleolus; max, maximum; med, medial; METh, metabolic equivalent hours; min, minutes; $m l$, millimetres; $N A$, not applicable; $n o$, number; $N R$, not reported; OM, outcome measure; $p t$, participant; $R$, right; reps, repetitions; $S C T$, stair-climb test; $S O B$, shortness of breath; $\mathrm{SpO}_{2}$, oxygen saturation; $U L$, upper limb; $V E$, minute ventilation; $V_{2}{ }_{2}$ oxygen uptake; $V O_{2} m a x$; maximal oxygen consumption; $y$ r, year published. 
Table 4 Criterion-concurrent validity, criterion-predictive validity and construct validity of outcome measures

\begin{tabular}{|c|c|c|c|c|}
\hline Author, yr & $\begin{array}{l}\text { Type of validity } \\
\text { and OM }\end{array}$ & Missing values & Comparator OM or predicted outcome & Validation results \\
\hline \multicolumn{5}{|l|}{ Functional capacity } \\
\hline Jones 2012 [40] & Crit-pred 6MWT & Nil & All-cause mortality & $\begin{array}{l}\text { Unadjusted } \mathrm{HR} \mathrm{p}=0.003 \text {; Compared to } 6 \mathrm{MWT} \\
<358.5 \mathrm{~m} \text { adjusted } \mathrm{HR}=0.61(95 \% \mathrm{Cl} 0.34-1.07) \\
\text { if } 6 \mathrm{MWT} 358.5-450 \mathrm{~m} \text {; Compared to } 6 \mathrm{MWT}<385.5 \mathrm{~m} \\
\text { adjusted } \mathrm{HR}=0.48(95 \% \mathrm{Cl} 0.24-0.93) \text { if } 6 \mathrm{MWT}>450 \mathrm{~m}\end{array}$ \\
\hline Pancieri 2010 [22] & Crit-pred 6MWT & $N R$ & $\begin{array}{l}\text { Predicted post-op } 6 \mathrm{MWT}=\text { pre-op } \\
6 \mathrm{MWT} \times(\mathrm{FS}-\text { resected FS }) \div \mathrm{FS}\end{array}$ & $r=0.40, p<0.01$ \\
\hline Kasymjanova 2009 [25] & Crit-pred 6MWT & 19pts dropped out & Survival & $\begin{array}{l}\text { Compared to } 6 \mathrm{MWT} \geq 400 \mathrm{~m} \text { mortality HR }=0.44 \\
(95 \% \mathrm{Cl} 0.23-0.83) \text { if } 6 \mathrm{MWT}<400 \mathrm{~m}, \mathrm{p}=0.001\end{array}$ \\
\hline \multirow[t]{4}{*}{ Mao 2007* Miller $2005[26,27]$} & Crit-pred; construct 6MWT & 3pts not complete Ax & 1. Development of RP & 1. $\mathrm{ROC}$ area under curve $=0.41, p=0.4$ \\
\hline & & & 2. $\mathrm{FEV}_{1}$ & 2. $r=0.53, p<0.001$ \\
\hline & & & 3. FVC & 3. $r=0.44, p=0.001$ \\
\hline & & & 4. DLCO & 4. $r=0.48, p<0.001$ \\
\hline \multirow[t]{4}{*}{ Saad 2007 [24] } & Crit-pred 6MWT & $\begin{array}{l}\text { 9pts died; 30pts not } \\
\text { complete Ax (rural) }\end{array}$ & $\begin{array}{l}\text { Predictors of improvement in } \\
\text { pre-op to } 180 \text { days-post-op: }\end{array}$ & 1. $\mathrm{GEE}=0.001, \mathrm{SE}=0.000, p=0.003$ \\
\hline & & & 1. SF-36 PF & 2. $\mathrm{GEE}=0.001, \mathrm{SE}=0.000, p=0.000$ \\
\hline & & & 2. SF-36 PR & 3. $\mathrm{GEE}=0.001, \mathrm{SE}=0.000, p=0.031$ \\
\hline & & & 3. SF-36 GH & \\
\hline Parsons 2003 [39] & Crit-pred 6MWT & 29pts & LOS out of hospital $<30$ days post-op & Not significant \\
\hline \multirow[t]{6}{*}{ Pierce 1994 [23] } & Crit-pred 6MWT & $N R$ & Post-op: & 1. $p<0.05$ \\
\hline & & & 1. respiratory failure & 2. $p>0.05$ \\
\hline & & & 2. surgical POC & 3. $p>0.05$ \\
\hline & & & 3. respiratory POC & 4. $p>0.05$ \\
\hline & & & 4. cardiac POC & 5. $p>0.05$ \\
\hline & & & 5. all POC & \\
\hline \multirow[t]{2}{*}{ Holden 1992 [41] } & Crit-pred 6MWT & 3pts not complete Ax & Survival $>90$ days post-op & $6 \mathrm{MWT}$ diff b/t groups with/without survival $p<0.05$ \\
\hline & & & & $\begin{array}{l}6 \mathrm{MWT}>1000 \text { feet }(305 \mathrm{~m}) \text { pre-op sensitivity } 100 \% \text {, } \\
\text { positive predictive value } 85 \% \text {, negative predictive } \\
\text { value } 100 \% \text { for survival }\end{array}$ \\
\hline \multirow[t]{5}{*}{ England 2012 [44] } & Construct ISWT & Nil & 1. P max monitor (insp mm strength) & 1. $r=0.42, p=0.01$ \\
\hline & & & 2. dynamometry (peripheral mm power) & 2. $r=0.39, p=0.01$ \\
\hline & & & 3. spirometry (\% predicted $\mathrm{FEV}_{1}$ ) & 3. $r=0.22, p=0.17$ \\
\hline & & & 4. spirometry (\% predicted FVC) & 4. $r=0.21, p=0.2$ \\
\hline & & & 5. CRDQ (mastery) & 5. $r=0.21, p=0.18$ \\
\hline
\end{tabular}


Table 4 Criterion-concurrent validity, criterion-predictive validity and construct validity of outcome measures (Continued)

\begin{tabular}{|c|c|c|c|c|}
\hline \multirow[t]{4}{*}{ Win 2006 [30] } & \multirow{4}{*}{$\begin{array}{l}\text { Crit-pred; crit-concurrent; } \\
\text { construct ISWT }\end{array}$} & \multirow[t]{4}{*}{ Nil } & 1. $C P E T\left(\mathrm{VO}_{2 \text { peak }}\right)$ & 1. $r=0.67, p<0.001$ \\
\hline & & & 2. CPET $\left(\mathrm{VO}_{2 \text { peak }} \%\right.$ predicted $)$ & 2. $r=0.30$ \\
\hline & & & 3. spirometry $\left(\mathrm{FEV}_{1}\right)$ & 3. $r=0.5$ \\
\hline & & & 4. 12 month survival & 4. $\mathrm{ROC}$ area $=0.7, p=0.003$ \\
\hline \multirow[t]{2}{*}{ Win 2004 [64] } & \multirow[t]{2}{*}{ Crit-pred, construct ISWT } & \multirow[t]{2}{*}{$8 p t s$} & $\begin{array}{l}\text { 1. poor surgical outcome (post-op death, } \\
\text { Ml, heart failure, resp failure, septicaemia, } \\
\text { pneumonia, cardiac arrthymia) }\end{array}$ & 1. $p=0.6$ between poor and sufficient outcome groups \\
\hline & & & 2. $\mathrm{FEV}_{1}$ & 2. $r=0.46$, not significant \\
\hline Brunelli 2012 [43] & Crit-pred SCT & 14 & Median survival and 5-year survival & Altitude $>18 \mathrm{~m}$ independent predictor: $\mathrm{HR}=0.5, \mathrm{p}=0.003$ \\
\hline \multirow[t]{2}{*}{ Brunelli 2010 [31] } & \multirow[t]{2}{*}{ Construct SCT } & \multirow[t]{2}{*}{ Nil } & \multirow{2}{*}{$\mathrm{SCT}\left(\mathrm{VO}_{2 \max }\right)$} & Altitude: correlation coefficient $=0.7, p<0.0001$ \\
\hline & & & & Speed of ascent: correlation coefficient $=0.47, p=0.005$ \\
\hline Pancieri 2010 [22] & Crit-pred SCT & NR & $\begin{array}{l}\text { Predicted post-op SCT }=\text { pre-op SCT } x \\
(\text { FS }- \text { resected FS }) \div F S\end{array}$ & $r=0.66, p<0.001$ \\
\hline \multirow[t]{2}{*}{ Brunelli 2008a [34] } & \multirow[t]{2}{*}{ Crit-pred SCT } & \multirow[t]{2}{*}{ Nil } & \multirow[t]{2}{*}{$\mathrm{POC}<30$ days post-op } & $\begin{array}{l}\text { Pre-op altitude: coefficient }=-0.05, \mathrm{OR}=0.95(95 \% \mathrm{Cl} 0.91-0.99) \\
\mathrm{SE}=0.02, \mathrm{p}=0.045\end{array}$ \\
\hline & & & & $\begin{array}{l}\mathrm{O}_{2} \text { desat }>4 \% \text { : coefficient }=0.56, \mathrm{OR}=1.8(95 \% \mathrm{Cl} 1-3), \mathrm{SE}=0.3 \\
\mathrm{p}=0.05\end{array}$ \\
\hline \multirow[t]{3}{*}{ Brunelli 2008b [33] } & \multirow[t]{3}{*}{ Crit-pred SCT } & \multirow[t]{3}{*}{ Nil } & 1. POC $<30$ days post-op & $\begin{array}{l}\text { 1. altitude: coefficient }=0.34, S E=0.2, O R=1.4(95 \% C l \text { 1.02-1.95), } \\
p=0.04\end{array}$ \\
\hline & & & 2. Death $<30$ days post-op & $\begin{array}{l}\text { 2. altitude: coefficient }=0.91, \mathrm{SE}=0.4, \mathrm{OR}=2.5(95 \% \mathrm{Cl} 1.1-5.5) \text {, } \\
\mathrm{p}=0.02\end{array}$ \\
\hline & & & 3. Post-op hospital costs & 3. altitude: coefficient $=2160.2$, SE $=573, p<0.001$ \\
\hline Koegelenberg 2008 [29] & Crit-concurrent SCT & Nil & CPET $\left(\mathrm{VO}_{2 \max }\right)$ & Altitude $r^{2}=0.06$, Speed of ascent $r^{2}=0.77$ (lung cancer only) \\
\hline Nikolic 2008 [36] & Crit-pred SCT & Nil & $\mathrm{POC}<30$ days post-op & $\begin{array}{l}\text { Best independent predictor }=\mathrm{SpO}_{2} \text { after } 40 \text { steps and } \mathrm{SCT} \\
\text { duration for lobectomy group }(60 \% \text { sensitivity, } 75 \% \text { specificity cut } \\
\text { off value } 1.09 \text { ) positive } \mathrm{LR}=2.4(95 \% \mathrm{Cl} 1.71-3.38) \text {, negative } \\
\mathrm{LR}=0.53(95 \% \mathrm{Cl} 0.38-0.76)\end{array}$ \\
\hline Brunelli 2007 [20] & Constuct SCT & 53pts at 3 months & Post-op SCT $\left(\mathrm{VO}_{2}\right.$ peak) & $\begin{array}{l}\text { Pre-op } \mathrm{SCT} \mathrm{VO} \mathrm{V}_{2} \text { peak directly associated with post-op SCT: } \\
\text { regression analysis lobectomy } \mathrm{F}=3.58, \mathrm{p}<0.01 \text {; pneumonectomy } \\
\mathrm{F}=3.53, \mathrm{p}<0.01\end{array}$ \\
\hline \multirow[t]{2}{*}{ Toker 2007 [37] } & \multirow[t]{2}{*}{ Crit-pred SCT } & \multirow[t]{2}{*}{ Nil } & \multirow[t]{2}{*}{ POC (cardiac or pulmonary) } & $\mathrm{SpO}_{2}$ pre-SCT: $\mathrm{OR}=0.74(95 \% \mathrm{Cl} 0.58-1.00), \mathrm{p}=0.001$ \\
\hline & & & & Change $\mathrm{SpO}_{2}$ pre to post-SCT: $\mathrm{OR}=1.59(95 \% \mathrm{Cl} 1.21-2.10), \mathrm{p}=0.018$ \\
\hline \multirow[t]{3}{*}{ Brunelli 2005 [42] } & \multirow[t]{3}{*}{ Crit-pred SCT } & \multirow[t]{3}{*}{ 13pts } & \multirow[t]{2}{*}{ 1. $\mathrm{POC}<30$ days post-op } & Inability to perform pre-op SCT: \\
\hline & & & & 1. $p=0.7$ \\
\hline & & & 2. Death $<30$ days post-op & 2. $\mathrm{OR}=0.20(95 \% \mathrm{Cl} 0.06-0.62), \mathrm{p}=0.005$ \\
\hline Brunelli 2004 [35] & Crit-pred SCT & 18pts & $\mathrm{POC}<30$ days post-op & $\begin{array}{l}\text { Lower altitude pre-op independent predictor: coefficient }=-0.18 \text {, } \\
p=0.0015\end{array}$ \\
\hline
\end{tabular}


Table 4 Criterion-concurrent validity, criterion-predictive validity and construct validity of outcome measures (Continued)

\begin{tabular}{|c|c|c|c|c|}
\hline Brunelli 2003 [21] & Crit-pred SCT & $\mathrm{Nil}$ & $\mathrm{O}_{2}$ desat during post-op SCT & $\begin{array}{l}\mathrm{O}_{2} \text { desat during pre-op SCT independent variable: regression } \\
\text { coefficient }=0.22, p=0.0004\end{array}$ \\
\hline \multirow[t]{2}{*}{ Parsons 2003 [39] } & \multirow[t]{2}{*}{ Crit-pred SCT } & \multirow[t]{2}{*}{ 29pts } & \multirow[t]{2}{*}{ LOS out of hospital $<30$ days post-op } & 1. longer LOS correlated with speed of ascent $r=0.34, p \leq 0.02$ \\
\hline & & & & 2. workload achieved predicted LOS out of hospital $r^{2}=0.130$ \\
\hline Brunelli 2002 [32] & Crit-pred SCT & $\mathrm{Nil}$ & $\mathrm{POC}<30$ days post-op & Altitude independent variable: $p=0.003$ \\
\hline Pate 1996 [38] & Crit-pred SCT & $\mathrm{Nil}$ & $\mathrm{POC}<30$ days post-op & $\begin{array}{l}\text { Significant difference in pre-op SCT between pt who did and } \\
\text { did not develop POC }\end{array}$ \\
\hline \multirow[t]{2}{*}{ Holden 1982 [41] } & \multirow[t]{2}{*}{ Crit-pred SCT } & \multirow[t]{2}{*}{$3 p t s$} & \multirow[t]{2}{*}{ Survival $>90$ days post-op } & $\mathrm{SCT}$ diff b/t groups with/without survival $p<0.05$ \\
\hline & & & & $\begin{array}{l}\text { SCT > 44steps pre-op positive predictive value } 91 \% \text {, negative } \\
\text { predictive value } 80 \% \text { for survival }\end{array}$ \\
\hline \multicolumn{5}{|l|}{ Physical activity } \\
\hline Maddocks 2012 [47] & Construct Acc & $\mathrm{Nil}$ & ECOG PS & $\begin{array}{l}\text { Statistically significant difference in mean daily step count, time } \\
\text { spent sitting/lying, upright, standing or stepping between } \\
\text { PS } 0,1 \text { and } 2 p<0.05 \text { but not mean daily sit-to-stand transitions }\end{array}$ \\
\hline \multirow{4}{*}{$\begin{array}{l}\text { Grutsch } 2011 \mathrm{a}, 2011 \mathrm{~b} ; \\
\text { Du-Quiton } 2010 \text { [48-50] }\end{array}$} & \multirow[t]{4}{*}{ Construct Acc } & \multirow{4}{*}{$\begin{array}{l}\text { 16pts Acc, } 16 \mathrm{pts} \\
\text { questionnaires }\end{array}$} & 1. HADS & 1. Outpt: depression and activity $r=-0.41, p=0.04$ \\
\hline & & & 2. Ferrans and Power QLI Cancer Version III & $\begin{array}{l}\text { 2. Daytime activity and QLI domains of health/functioning } \\
r=0.51, p<0.01 ; \text { social/economic } r=0.38, p=0.048 \text {; } \\
\text { psychological/spiritual } r=0.45, p=0.02 ; \text { family } r=0.45 \\
p=0.02 ; \text { overall QLI } r=0.57, p<0.01\end{array}$ \\
\hline & & & 3. EORTC & 3. Inpt: daytime activity and loss of appetite $r=-0.41, p=0.005$ \\
\hline & & & 4. PSQI & 4. Outpt: lower sleep medication use and activity $r=-0.58, p<0.01$ \\
\hline \multirow[t]{4}{*}{ Novoa 2011 [51] } & \multirow[t]{4}{*}{ Construct Pedom } & \multirow{4}{*}{$\begin{array}{l}13 \text { pts - unable to } \\
\text { perform exercise test }\end{array}$} & \multirow[t]{4}{*}{ CPET $\left(\mathrm{VO}_{2 \max }\right)$} & Mean daily total steps $r=0.4$ \\
\hline & & & & Mean daily aerobic steps $r=0.16$ \\
\hline & & & & Mean daily time of aerobic capacity $r=0.11$ \\
\hline & & & & Mean daily distance walked $r=0.44$ \\
\hline Maddocks 2010 [46] & Construct Acc & 2 pts withdrawn & $\begin{array}{l}\text { Estimated EE (stepping and non-stepping) } \\
\text { measured from acc }\end{array}$ & Non-stepping EE and daily step count $r=-0.91, p<0.01$ \\
\hline \multicolumn{5}{|l|}{ Muscle strength } \\
\hline \multirow[t]{2}{*}{ Brown 2005 [53] } & \multirow[t]{2}{*}{ Construct CST } & \multirow[t]{2}{*}{ Nil } & 1. KPS & 1. $r^{2}=0.565, p<0.001$ (ca group) \\
\hline & & & 2. FACIT-fatigue & 2. with incr fatigue, lower CST $p<0.01$ (ca group) \\
\hline
\end{tabular}

Abbreviations: 6MWT, six minute walk test; $95 \% \mathrm{Cl}$, 95\% confidence intervals; acc, accelerometery; $A x$, assessment; b/t, between; ca, cancer; CPET, cardio-pulmonary exercise testing; CRDQ, Chronic Respiratory Disease Questionnaire; crit, criterion; CST, chair-stand test; desat, desaturation; diff, difference; DLCO, diffused capacity for carbon monoxide; ECOG, Eastern Cooperative Oncology Group; EE, energy expenditure; EORTC,

European Organisation for Research and Treatment of Cancer quality of life questionnaire; FACIT-fatigue, Functional Assessment of Chronic Illness Therapy- Fatigue scale; FEV ${ }_{1}$, force expired volume in one second; FS, lung functioning segments; FVC, forced vital capacity; GEE, generalized estimation equations; HADS, Hospital Anxiety and Depression Scale; HR, hazard ratio; insp, inspiratory; inpt, inpatient; ISWT, incremental-shuttle walk test; $K P S$, Karnofsky performance status; $L O S$, length of stay; $L R$, likelihood ratio; $m$, meters; max, maximum; $M I$, myocardial infarction; $m m$, muscle; $N R$, not reported; OM, outcome measure; $O R$, odds ratio; outpt, outpatient; $P$, pressure; pedom, pedometer; $P O C$, post-operative complication; post-op, post-operative; pre-op, pre-operative; pred, predictive; PS, performance status; $p t$, participant; $P S Q I$, Pittsburgh Sleep Quality Index; QLI, Quality of Life Index; $r$, correlation coefficient; ROC, received operating characteristic curve; SCT, stair-climb test; SE, standard error; SF-36, Short Form 36 physical functioning/physical role/general health domain; resp, respiratory; $R P$, radiation pneumonitis; $R x$, treatment; $\mathrm{SpO}_{2}$, oxygen saturation; $\mathrm{VO}_{2 \text { max }}$, maximum oxygen consumption; $V_{2}$ peak, peak oxygen consumption; $y r$, year published.

* results presented from most recent publication. 
'excellent' or 'good' on 12/29 occasions. No studies evaluating reliability scored 'excellent' or 'good' (Table 5). The worst performing area for validity studies was design requirements (lack of a priori hypotheses formed) and for reliability studies was design requirements (small sample size).

\section{Study results}

Study results are summarised in Table 1 and the sections below. The stair-climbing test, six-minute walking test
(6MWT) and incremental-shuttle walk test (ISWT) performed the best out of the 13 tests reviewed, primarily due to lack of studies investigating measurement properties of the other 10 tests (Table 1 ).

Functional capacity The 6MWT, twelve-minute walking test (12MWT), ISWT, endurance-shuttle walking test (ESWT) and stair-climbing test are field tests reflecting functional capacity. No studies investigated

Table 5 Methodological quality of included studies - part two

\begin{tabular}{|c|c|c|c|c|c|}
\hline Author, yr & Reliability & Measurement error & Hypothesis testing & Criterion validity & Responsiveness \\
\hline \multicolumn{6}{|l|}{ Functional capacity } \\
\hline Brunelli 2012 [43] & $x$ & $x$ & $x$ & Fair & $x$ \\
\hline England 2012 [44] & $x$ & $x$ & Fair & $x$ & $x$ \\
\hline Jones 2012 [40] & $x$ & $x$ & $x$ & Excellent & $x$ \\
\hline Brunelli 2010 [31] & $x$ & $x$ & Excellent & $x$ & $x$ \\
\hline Pancieri 2010 [22] & $x$ & $x$ & $x$ & Fair & $x$ \\
\hline Kasymjanova 2009 [25] & $x$ & $x$ & $x$ & Fair & $x$ \\
\hline Brunelli 2008a [34] & $x$ & $x$ & $x$ & Excellent & $x$ \\
\hline Brunelli 2008b [33] & $x$ & $x$ & $x$ & Excellent & $x$ \\
\hline Koegelenberg 2008 [29] & $x$ & $x$ & $x$ & Fair & $x$ \\
\hline Nikolic 2008 [36] & $x$ & $x$ & $x$ & Poor & $x$ \\
\hline Brunelli 2007 [20] & $x$ & $x$ & Fair & $x$ & $x$ \\
\hline Mao 2007 [26] & $x$ & $x$ & Fair & Good & $x$ \\
\hline Saad 2007 [24] & $x$ & $x$ & $x$ & Poor & $x$ \\
\hline Toker 2007 [37] & $x$ & $x$ & $x$ & Excellent & $x$ \\
\hline Win 2006 [30] & $x$ & $x$ & Fair & Good & $x$ \\
\hline Brunelli 2005 [42] & $x$ & $x$ & $x$ & Excellent & $x$ \\
\hline Brunelli 2004 [35] & $x$ & $x$ & $x$ & Good & $x$ \\
\hline Win 2004 [64] & $x$ & $x$ & Poor & Poor & $x$ \\
\hline Brunelli 2003 [21] & $x$ & $x$ & $x$ & Excellent & $x$ \\
\hline Parsons 2003 [39] & $x$ & $x$ & $x$ & Good & $x$ \\
\hline Brunelli 2002 [32] & $x$ & $x$ & $x$ & Excellent & $x$ \\
\hline Pate 1996 [38] & $x$ & $x$ & $x$ & Poor & $x$ \\
\hline Pierce 1994 [23] & $x$ & $x$ & $x$ & Poor & $x$ \\
\hline Holden 1992 [41] & $x$ & $x$ & $x$ & Poor & $x$ \\
\hline \multicolumn{6}{|l|}{ Physical activity } \\
\hline Maddocks 2012 [47] & $x$ & $x$ & Poor & $x$ & $x$ \\
\hline Novoa 2011 [51] & $x$ & $x$ & Poor & $x$ & $x$ \\
\hline Grutsch 2011a, 2011b; Du-Quiton 2010 [48-50] & $x$ & $x$ & Fair & $x$ & $x$ \\
\hline Maddocks 2010 [46] & $x$ & $x$ & Fair & $x$ & $x$ \\
\hline Muscle strength & & & & $x$ & $x$ \\
\hline Trutschnigg 2008 [28] & Poor (intra-r) & $x$ & $x$ & $x$ & $x$ \\
\hline Brown 2005 [53] & $x$ & $x$ & Fair & $x$ & $x$ \\
\hline Knols 2002 [52] & Fair (inter-r) & Fair & $x$ & $x$ & $x$ \\
\hline
\end{tabular}

Abbreviations: inter-r, inter-rater reliability; intra-r, intra-rater reliability; yr, year published. $\mathrm{x}=$ not assessed 
inter or intra-rater reliability, measurement error or responsiveness of these tests in participants with NSCLC.

The criterion-concurrent validity of the ISWT and stairclimbing test against the gold standard cardio-pulmonary exercise test (CPET) was reported by three studies (Table 4) [29-31]. The ISWT was validated against CPET $\left(\mathrm{VO}_{2}\right.$ peak) with strong correlation $(\mathrm{r}=0.67)$ [30]. The stairclimbing test (ascent speed) was validated against CPET (maximum oxygen consumption $\mathrm{VO}_{2} \max$ ) with strong correlation $\left(\mathrm{r}^{2}=0.77\right)$ [29].

The criterion-predictive validity of the 6MWT, ISWT and stair-climbing test were reported and these instruments were shown to predict post-operative outcomes (studies $\mathrm{n}=12$ ) [20-24,32-38], post-operative length of hospital stay $(n=1)$ [39] and survival $(n=8)$ (Table 4) [23,25,30,33,40-43]: Pre-operative stair-climbing test was a predictor for post-operative complications when using variables: test duration [36], oxygen saturation [34,36,37] or altitude [32-35,38]. Pre-operative 6MWT was a predictor for post-operative respiratory failure $(\mathrm{p}<0.05)$ [23]. Pre-operative stair-climbing test was a predictor for post-operative length of stay $(\mathrm{r}=0.34)$ [39] and hospital cost (coefficient=2160.2) [33]; and 6MWT was a predictor for post-operative health related quality of life (HRQoL) physical domains (GEE=0.001) [24]. The 6MWT was shown in two papers to predict survival in advanced NSCLC (hazard ratios=0.44 [25] and 0.48 [40]). With every $50 \mathrm{~m}$ improvement in 6MWT, survival improved by $13 \%$ [40] and patients walking $\geq 400 \mathrm{~m}$ pre-chemotherapy had greater survival time [25]. In the post-operative population survival was predicted by pre-operative ISWT (area under the ROC curve=0.7) [30]; stair-climbing test (steps climbed) $(\mathrm{p}<0.05)$ [41]; stair-climbing test (altitude) (coefficient $=0.91$ [33]; hazard ratio=0.5 [43]) and inability to perform stair-climbing test (odds ratio=0.2) [42]. A pre-operative stair-climbing test result of $>44$ steps predicted post-operative survival at 30 days (positive predictive value $=91 \%$, negative predictive value $=80 \%$ ) [41].

Three studies reported on the construct validity of the 6MWT and ISWT: The 6MWT was validated against respiratory function tests (forced expired volume in onesecond) with strong correlation $(r=0.53)$ [26]. The ISWT was validated with moderate correlation against inspiratory muscle strength $(\mathrm{r}=0.42)$ [44] and isokinetic muscle dynamometry ( $\mathrm{r}=0.39)$ (Table 4) [44].

Physical activity No studies validated accelerometers or pedometers against the gold standard measure of physical activity (direct calorimetry) [45] or investigated reliability, measurement error or responsiveness. Four studies investigated construct validity (Table 4): The ActivPAL ${ }^{\mathrm{TM}}$ accelerometer (step count) was validated against ActivPAL $^{\mathrm{TM}}$ (estimated energy expenditure) with strong correlation $(\mathrm{r}=-0.91)$ [46] and Eastern Cooperative Oncology Group
(ECOG) Performance-Scale $(\mathrm{p}<0.05)$ [47]. The Actigraph (accelerations/minute) was validated with medium correlation against the Hospital Anxiety and Depression Scale (depression) ( $\mathrm{r}=-0.41)$ [48], the Ferrans and Power Quality of Life Index Cancer-Version III (HRQoL) ( $\mathrm{r}=0.38-0.57$ ) [49], the European Organisation for Research and Treatment of Cancer quality of life questionnaire (loss of appetite) ( $\mathrm{r}=-0.41)$ [49]; and with strong correlation against the Pittsburgh Sleep Quality Index (sleep medication use) $(\mathrm{r}=-0.58)$ [50]. The OMROM Walking Style Pro ${ }^{\circledR}$ pedometer (distance walked) was validated against CPET $\left(\mathrm{VO}_{2} \mathrm{max}\right)$ with moderate correlation $(\mathrm{r}=0.4)$ [51].

Muscle strength Only two studies investigated muscle strength test reliability (Table 1; Additional file 3): The inter-rater reliability of the MFB50K pulley-gauge hand-held dynamometer (HHD) (elbow/knee extension) was very good (ICC $=0.90,0.96$ respectively), however measurement error between examiners was large (SEM=10.6, 19.8 respectively), as was the smallest detectable difference (SDD $=29.4,54.8$ respectively) (Additional file 4) [52]. The Jamar hand-grip dynamometer (HGD) (gripstrength) intra-rater reliability percent coefficient of variation was 6.3, which was better than that demonstrated for HGD with Biodex attachment (\%CV16.7) (Table 1; Additional file 3) [28].

No tests measuring muscle strength were validated against the gold standard measure (isokinetic dynamometry). Construct validity was reported for the chair-stand test with a moderate correlation against Karnofsky Performance Status $\left(\mathrm{r}^{2}=0.56\right)($ Table 4$)$ [53].

\section{Discussion}

This review focused on three commonly assessed outcomes (functional capacity, physical activity and muscle strength) used in the NSCLC literature [3]. Tests used to evaluate the effectiveness of exercise in patients with NSCLC must be reliable and responsive to change in the outcome of interest, regardless of the cancer stage of participants and therefore understanding how different NSCLC stages respond to the outcome measures is vital. Standardised measures allow generalizability of study results across trials, which is important in NSCLC, given the poor participant consent/retention rate [54] and mortality rate. The gold standard measurement of functional capacity, physical activity and muscle strength require laboratory tests which have significant limitations for use in exercise-based NSCLC research trials. CPET (functional capacity) [7], direct calorimetry (physical activity) and isokinetic dynamometry (muscle strength) require expensive equipment, advanced monitoring and experienced technicians. Whilst limited studies have reported CPET to be safe and feasible in NSCLC [55], field tests which be performed reliably in clinical settings may reduce research costs, participant 
burden and drop-out rates. This review demonstrated the use of 13 different field tests and, although a number of studies investigated the validity of outcome measures in NSCLC, only two studies investigated reliability, with no study investigating test responsiveness. Further studies are needed to establish measurement properties of standardised field tests for individuals with NSCLC to allow the most appropriate choice of test when designing research trials.

Functional capacity was the most common outcome of interest in this review, with the 6MWT most commonly used. Search 1 retrieved 38 studies utilising the 6MWT in NSCLC and Search 2 retrieved seven studies investigating 6 MWT measurement properties. Only 51\% $(n=17 / 33)$ of studies published after 2002, using the 6MWT in Search 1, referenced the American Thoracic Society guidelines in their methodology [56]. Three studies referenced the guidelines but stated they performed only one 6MWT during a testing session. Two tests have been shown to enhance reliability in other populations, with reports demonstrating the second 6MWT increases by $9-15 \mathrm{~m}$ $[56,57]$. The encouragement used in the 6MWT in part one studies was variable. No studies identified in part two of this review analysed the reliability of the 6MWT. Similarly, in Search 1, 14 studies used the 6MWT to evaluate the benefit of exercise intervention over time, however no studies in Search 2 investigated the responsiveness of the 6MWT in any stage of NSCLC. In comparison, there has been a substantial amount of work regarding the criterionpredictive validity of the $6 \mathrm{MWT}$ in patients with NSCLC. Results demonstrated the $6 \mathrm{MWT}$ was predictive for post-operative complications, HRQoL and survival. The 6MWT has not been validated against CPET in NSCLC, however it has been validated against CPET in populations with cardiorespiratory disease with moderate correlations ( $r=0.51-0.93)$ [58-61]. Given the frequent use of the 6MWT, establishing reliability, measurement error, minimal clinically important difference, responsiveness and validating the 6MWT against CPET in NSCLC should be a priority.

In Search 1 the ISWT was used in six studies involving participants with NSCLC and twice this was to evaluate the benefit of exercise $[62,63]$. Only fifty percent of the studies described how the participant was monitored during the test $[30,44,64]$, however all studies referenced their procedure, most $(n=5 / 6,83 \%)$ referencing the original protocol when the test was created [65]. The ISWT was only performed once during the testing session across all studies excluding one. Given no studies in Search 2 investigated the reliability of this test, similar to the case with the 6MWT, further research needs to investigate the best method for completing it in NSCLC to determine if a familiarisation effect is present.

The 12MWT and the ESWT have been infrequently used in studies of NSCLC and neither test was investigated regarding its measurement properties in NSCLC. Currently the alternative 6MWT and ISWT appear to be better choices of tests until further research is completed.

Search 1 identified 21 studies utilising the stair-climbing test in NSCLC, all in pre-lung resection candidates. No studies have used the stair-climbing test to evaluate exercise intervention. Currently there is no gold standard method to perform the stair-climbing test. Published studies used variable instructions, encouragement, monitoring and experience of assessors. Some authors reported the number of steps/altitude whilst others reported test duration. Results of Search 2 consistently demonstrated the stair-climbing test to be valuable in the pre-operative evaluation of lung resection candidates, with the stairclimbing test providing prediction validity with regard to post-operative complications, length of stay, mortality and hospital cost. The stair-climbing test has also been validated against the gold standard (CPET). No studies evaluated reliability; measurement error or responsiveness in NSCLC and therefore it is currently not known if this is a suitable test to evaluate exercise interventions, especially in post-operative and chemo-radiation cohorts.

Search 1 demonstrated that physical activity has been measured in participants with NSCLC using accelerometers and pedometers. Search 2 showed that accelerometers and pedometers have not been validated against the gold standard measure (direct calorimetry) in NSCLC. Direct calorimetry has limitations and accelerometers are commonly the preferred method to measure physical activity $[66,67]$. However, accelerometers and pedometers are limited in that they rely on participant compliance. In the NSCLC literature, few studies are conducted measuring physical activity levels and even fewer studies have investigated the measurement properties associated with tests.

Muscle strength was measured using five different tests by 17 studies in sSarch 1 . Search 2 retrieved three studies evaluating measurement properties of only three of the five instruments. All three studies were conducted with mixed cancer cohorts and the methodological quality of each study was 'poor' or 'fair': therefore results need to be interpreted with caution. Hand dynamometry was the most commonly used instrument to assess muscle strength in part one studies. Two hand-dynamometry devices were tested for reliability however results were not strong enough to recommend use of a particular device. Whilst both HHD and HGD have been shown to be reliable and valid in many patient populations, further research needs to be performed in NSCLC [68-70]. Manual muscle testing is often considered to be qualitative and frequently performed in profoundly weak populations such as those with critical illness [71,72]. Four studies in Search 1 used MMT to measure upper-body strength on repeated occasions however the measurement properties have not been established. This review demonstrated that 
HHD, HGD, MMT, one-repetition maximum and the chair-stand test have been used in NSCLC, however there is currently insufficient research to support the use of one measure over another.

\section{Limitations}

To minimise risk of selection bias two independent reviewers were utilised. In Search 2 articles were excluded if cancer type was unconfirmed. There is a risk of publication bias, where studies which have found poor measurement properties have not been published. Given that registration of studies evaluating measurement properties is not standard practice, the extent of this is unknown [8].

The COSMIN checklist was not completed in its entirety and may have also under-estimated methodological quality because the rating of each item was determined using the lowest score rather than the average or highest score.

Due to the small number of studies evaluating measurement properties of the included outcome measures in cohorts with only NSCLC participants, this review included studies with mixed cancer types (providing at least five participants had NSCLC). Different cancer types are associated with heterogeneous symptom profiles (for example dyspnoea and pain), gas exchange and exercise capacity. Therefore findings from the studies with mixed cancer types must be interpreted with caution when extrapolated for use in NSCLC. Additionally there was heterogeneity with regards to the participants in the included studies (particularly age and treatment exposure) (Table 2). This may explain, in part, the variance in data obtained and large standard deviations reported by individuals studies (Additional file 4) because age, comorbidities (such as COPD) and treatment (such as chemotherapy) directly impact exercise capacity and performance as well as the disease of NSCLC.

\section{Conclusion}

Measurements of functional capacity, physical activity and muscle strength are commonly used as outcomes for individuals with NSCLC participating in exercise trials. The 6MWT, 12MWT, ISWT, ESWT and stairclimbing test have been used to assess functional capacity in NSCLC. Only two tests (ISWT and stair-climb test) were validated against CPET, the gold standard measure of functional capacity. Physical activity has been measured using accelerometers and pedometers: there was some evidence for construct validity but neither had been validated against the gold standard or tested for reliability. Muscle strength has been measured using HHD, HGD, manual muscle test, 1RM and the chair-stand test. Only two strength measures were tested for their reliability in NSCLC, and there was insufficient evidence to support the use of one strength measure over another. Responsiveness and minimal important clinical difference was not established for any of the 13 tests. Currently there is an important gap in the literature regarding the measurement properties of commonly used tests in NSCLC and further research needs to be conducted in this area to improve the clinical use and applicability of these tests in patients with NSCLC.

\section{Additional files}

\begin{abstract}
Additional file 1: Flow diagram of outcome measures selection process - Search 1 [11]. Abbreviations: Ax, assessment; CINAHL,

Cumulative Index to Nursing and Allied Health Literature; DARE, Database of Abstracts and Reviews of Effects; EMBASE, the Excerpta Medica Database; FT, full text; n, number; NSCLC, non-small cell lung cancer; OM, outcome measure; PEDRO, Physiotherapy Evidence Database; PROM, patient reported outcome measure.
\end{abstract}

Additional file 2: Search strategy - Search 2 [17]. Abbreviations: CINAHL, Cumulative Index to Nursing and Allied Health Literature; EMBASE, the Excerpta Medica Database; MESH, Medical Subject Heading Indexing.

Additional file 3: Interpretability. Abbreviations: 6MWT, six minutewalk test; acc, accelerations; chemo, chemotherapy; CST, chair-stand test; E1, examiner one; E2, examiner two; Elb, elbow; E, extension; ECOG, Eastern Cooperative Oncology Group; ft, feet; gp, group; HGS, hand grip strength; hrs, hours; inpt, inpatients; IQR, inter-quartile range; ISWT, incremental-shuttle walk test; kg, kilogram; lbs, pounds; m, meters; MIC, minimal important change; min, minutes; $\mathrm{ml}$, millilitres; $\mathrm{N}$, newtons; outpt, outpatient; $\mathrm{O}_{2}$ desat, oxygen desaturation; POC, post-operative complication; post-op, post-operative; pre-op, pre-operative; PS, performance status; RT, radiotherapy; s, seconds; SCT, stair-climb test; SD, standard deviation; SDD, smallest detectable difference; $V_{2}$ peak, peak oxygen consumption; yr, year published.* results presented from most recent publication.

Additional file 4: Inter-rater reliability, intra-rater reliability and measurement error associated with outcome measures.

Abbreviations: 95\% Cl, 95\% confidence intervals;\%CV, percent coefficient of variation; b/t, between; E, extension; Elb, elbow; HGD, hand-grip dynamometry; HHD, hand-held dynamometry; ICC, intraclass correlation coefficient; mean diff, mean difference for repeated measures; min, minutes, OM, outcome measure; NR, not reported; SEM, standard error of measurement.

Competing interest

The authors declare that they have no competing interests.

\section{Authors' contribution}

CG participated in the design of the protocol, contributed to establishment of search terms, performed database searching, reviewed articles for inclusion from Search 1 and 2 (as first independent reviewer), performed quality appraisal (as first independent quality assessor) and drafted the manuscript. CMcD participated in the design of the protocol, contributed to the background literature search and manuscript preparation. SP reviewed articles for inclusion from Search 1 and 2 (as second independent reviewer), cross checked extracted data and contributed to manuscript preparation. CO contributed to establishment of search terms, development of data extraction forms, performed quality appraisal (as second independent quality assessor) and contributed to manuscript preparation. LD participated in the design of the protocol, background literature search, contributed to the development of the search strategy, reviewed articles for inclusion from Search 1 and 2 (as third independent reviewer) and contributed to the manuscript preparation. All authors read and approved the final manuscript.

\section{Acknowledgements}

The authors would like to acknowledge the authors/investigators from the included studies in this systematic review who willingly provided additional clarification information regarding their studies. The authors 
would also like to acknowledge $\operatorname{Dr}$ CB Terwee for her advice regarding the COSMIN4-point checklist.

CG was supported by an Australian Post-graduate Award Ph.D. student scholarship. There was no further source of funding or sponsorship for this systematic review.

\section{Author details}

'Department of Physiotherapy, School of Health Sciences, The University of Melbourne, Melbourne, Victoria, Australia. ${ }^{2}$ Institute for Breathing and Sleep, Melbourne, Victoria, Australia. ${ }^{3}$ Department of Respiratory and Sleep Medicine, Austin Health, Melbourne, Victoria, Australia. ${ }^{4}$ Department of Physiotherapy, Austin Health, Melbourne, Victoria, Australia.

Received: 4 October 2012 Accepted: 7 March 2013

Published: 20 March 2013

\section{References}

1. Jones $L$, Eves $N$, Haykowsky $M$, et al: Exercise intolerance in cancer and the role of exercise therapy to reverse dysfunction. Lancet Oncol 2009, 10:598-605.

2. Tanaka K, Akechi T, Okuyama T, et al: Impact of dyspnea, pain, and fatigue on daily life activities in ambulatory patients with advanced lung cancer. J Pain Symptom Manage 2002, 23:417-23.

3. Granger C, McDonald C, Berney S, et al: Exercise intervention to improve exercise capacity and health related quality of life for patients with Nonsmall cell lung cancer: a systematic review. Lung Cancer 2011, 72:139-53.

4. Fleg J, Pina I, Balady G, et al: Assessment of functional capacity in clinical and research applications - an advisory from the committee on exercise, rehabilitation, and prevention, council on clinical cardiology, American heart association. Circulation 2000, 102:1591-1597.

5. Caspersen C: Physical Activity, exercise and physical fitness: definitions and distinctions for health related research. Publ Health Rep 1985, 100:126-131.

6. Bohannon RW: Quantitative testing of muscle strength: issues and practical options for the geriatric population. Top Geriatr Rehabil 2002, 18:1-17.

7. Jones L, Eves N, Mackey J, et al: Safety and feasibility of cardiopulmonary exercise testing in patients with advanced cancer. Lung Canc 2007, 55:225-32.

8. de Vet $\mathrm{H}$, Terwee $\mathrm{C}$, Mokkink $\mathrm{L}$, et al: Measurement in Medicine - A Practical Guide. Cambridge: Cambridge University Press; 2011.

9. Portney L: Foundations of clinical research applications to practice. In . 2nd edition Edited by Watkins MP. Upper Saddle River, NJ: Prentice Hall; 2000.

10. Mokkink $L$, Terwee $C$, Knol D, et al: The COSMIN checklist for evaluating the methodological quality of studies on measurement properties: a clarification of its content. BMC Med Res Methodol 2010, 10:22-22.

11. Liberati A, Altman D, Tetzlaff J, et al: The PRISMA statement for reporting systematic reviews and meta-analyses of studies that evaluate healthcare interventions: explanation and elaboration. Br Med J 2009, 339:37.

12. NHMRC. National Health Medical Research Council: NHMRC additional levels of evidence and grades for recommendations for developers of guidelines. 2009. [cited 20128 July]; Available from: http://www.nhmrc.gov.au/ guidelines/developers.htm.

13. National Cancer Institute: US National Institutes of Health, Non-Small Cell Lung Cancer Treatment PDQ Summary. 2012. 15/10/12]; Available from: http://www.cancer.gov/cancertopics/pdq/treatment/non-small-cell-lung/ HealthProfessional/page1.

14. Terwee C, COSMIN group: An overview of systematic reviews of measurement properties of measurement instruments that intend to measure (aspects of) health status or (health-related) quality of life. 2012. [cited 2012 10.05]; Available from: http://www.cosmin.nl/the-cosmin-checklist_8_5.html.

15. National Institute for Health Research: International Prospective Register of Systematic Reviews (PROSPERO). 2012. [cited 2012 10.05]; Available from: http://www.crd.york.ac.uk/prospero/.

16. Sim J, Wright C: The kappa statistic in reliability studies: Use, interpretation, and sample size requirements. Phys Ther 2005, 85:257-268.

17. Terwee C, Jansma E, Riphagen I, et al: Development of a methodological PubMed search filter for finding studies on measurement properties of measurement instruments. Qual Life Res 2009, 18:1115-1123.

18. Terwee $C$, Mokkink $L$, Knol D, et al: Rating the methodological quality in systematic reviews of studies on measurement properties: a scoring system for the COSMIN checklist. Qual Life Res 2012, 21:651.
19. Terwee C, Bouwmeester W, van Elsland S, et al: Instruments to assess physical activity in patients with osteoarthritis of the hip or knee: a systematic review of measurement properties. Osteoarthr Cartil 2011, 19:620-33.

20. Brunelli $A$, Xiume $F$, Refai $M$, et al: Evaluation of expiratory volume, diffusion capacity, and exercise tolerance following major lung resection: a prospective follow-up analysis. Chest 2007, 131:141-7.

21. Brunelli A, Al Refai M, Monteverde $M$, et al: Predictors of exercise oxygen desaturation following major lung resection. Eur I Cardiothorac Surg 2003, 24:145-8.

22. Pancieri M, Cataneo D, Montovani J, et al: Comparison between actual and predicted postoperative stair-climbing test, walk test and spirometric values in patients undergoing lung resection. Acta Cir Bras 2010, 25:535-540.

23. Pierce R, Copland J, Sharpe K, et al: Preoperative risk evaluation for lung cancer resection: predicted postoperative product as a predictor of surgical mortality. Am J Respir Crit Care Med 1994, 150:947-55.

24. Saad I, Botega N, Toro I: Predictors of quality-of-life improvement following pulmonary resection due to lung cancer. Sao Paulo Med J 2007, 125:46-49.

25. Kasymjanova G, Correa J, Kreisman $\mathrm{H}$, et al: Prognostic value of the six-minute walk in advanced non-small cell lung cancer. J Thorac Oncol 2009, 4:602-7.

26. Mao J, Zhang J, Zhou S, et al: Updated assessment of the six-minute walk test as predictor of acute radiation-induced pneumonitis. Int J Radiat Oncol Biol Phys 2007, 67:759-67.

27. Miller K, Kocak Z, Kahn D, et al: Preliminary report of the 6-minute walk test as a predictor of radiation-induced pulmonary toxicity. IJROBP 2005, 62:1009-1013

28. Trutschnigg B: Precision and reliability of strength (Jamar vs. Biodex handgrip) and body composition (dual-energy X-ray absorptiometry vs. bioimpedance analysis) measurements in advanced cancer patients. Appl Physiol Nutr Met 2008, 33:1232-1239.

29. Koegelenberg C, Diacon A, Irani S, et al: Stair climbing in the functional assessment of lung resection candidates. Respiration 2008, 75:374-379.

30. Win T, Jackson A, Groves A, et al: Comparison of shuttle walk with measured peak oxygen consumption in patients with operable lung cancer. Thorax 2006, 61:57-60.

31. Brunelli $A$, Xiumé $F$, Refai $M$, et al: Peak oxygen consumption measured during the stair-climbing test in lung resection candidates. Respiration 2010, 80:207-211.

32. Brunelli $\mathrm{A}$, Al Refai $\mathrm{M}$, Monteverde $\mathrm{M}$, et al: Stair climbing test predicts cardiopulmonary complications after lung resection. Chest 2002, 121:1106-1109.

33. Brunelli A, Refai $M$, Xiume $F$, et al: Performance at symptom-limited stairclimbing test is associated with increased cardiopulmonary complications, mortality, and costs after major lung resection. Ann Thorac Surg 2008, 86:240-247. discussion 247-248.

34. Brunelli A, Refai M, Xiume F, et al: Oxygen desaturation during maximal stair-climbing test and postoperative complications after major lung resections. Eur J Cardiothorac Surg 2008, 33:77-81.

35. Brunelli A, Monteverde M, Al Refai M, et al: Stair climbing test as a predictor of cardiopulmonary complications after pulmonary lobectomy in the elderly. Ann Thorac Surg 2004, 77:266-270.

36. Nikolic I, Majeric-Kogler V, Plavec D, et al: Stairs climbing test with pulse oximetry as predictor of early postoperative complications in functionally impaired patients with lung cancer and elective lung surgery: prospective trial of consecutive series of patients. Croat Med J 2008, 49:50-7.

37. Toker A, Ziyade S, Bayrak Y, et al: Prediction of cardiopulmonary morbidity after resection for lung cancer: Stair climbing test complications after lung cancer surgery. Thorac Cardiovasc Surg 2007, 55:253-256.

38. Pate $\mathrm{P}$, Tenholder M, Griffin J, et al: Preoperative assessment of the highrisk patient for lung resection. Ann Thorac Surg 1996, 61:1494-500.

39. Parsons J, Johnston M, Slutsky A: Predicting length of stay out of hospital following lung resection using preoperative health status measures. Qual Life Res 2003, 12:645-54.

40. Jones L, Hornsby W, Goetzinger A, et al: Prognostic significance of functional capacity and exercise behavior in patients with metastatic non-small cell lung cancer. Lung Cancer 2012, 76:248-252.

41. Holden D, Rice T, Stelmach K, et al: Exercise testing, 6-min walk and stair climb in the evaluation of patients at high-risk for pulmonary resection. Chest 1992, 102:1774-1779.

42. Brunelli A, Sabbatini A, Xiume F, et al: Inability to perform maximal stair climbing test before lung resection: a propensity score analysis on early outcome. Eur J Cardiothorac Surg 2005, 27:367-72. 
43. Brunelli A, Pompili C, Berardi R, et al: Performance at Preoperative StairClimbing Test Is Associated With Prognosis After Pulmonary Resection in Stage I Non-Small Cell Lung Cancer. Ann Thorac Surg 2012, 93(6):1796-1801.

44. England R, Maddocks M, Manderson C, et al: Factors influencing exercise performance in thoracic cancer. Respir Med 2012, 106(2):294-299.

45. Vanhees $L$, Lefevre J, Philippaerts $R$, et al: How to assess physical activity? How to assess physical fitness? Eur J Cardiovasc Prev Rehabil 2005, 12:102-114.

46. Maddocks M: Physical activity level as an outcome measure for use in cancer cachexia trials: a feasibility study. Support Care Canc 2010, 18:1539-1544.

47. Maddocks M, Wilcock A: Exploring physical activity level in patients with thoracic cancer: implications for use as an outcome measure. Support Care Canc 2012, 20:1113-6.

48. Du-Quiton J, Wood P, Burch J, et al: Actigraphic assessment of daily sleepactivity pattern abnormalities reflects self-assessed depression and anxiety in outpatients with advanced non-small cell lung cancer. Psychooncology 2010, 19:180-189.

49. Grutsch J, Ferrans C, Wood P, et al: The association of quality of life with potentially remediable disruptions of circadian sleep/activity rhythms in patients with advanced lung cancer. BMC Canc 2011, 11:193.

50. Grutsch J, Wood P, Du-Quiton J, et al: Validation of actigraphy to assess circadian organization and sleep quality in patients with advanced lung cancer. J Circadian Rhy 2011, 9:4-4.

51. Novoa N, Varela G, Jimenez M, et al: Value of the average basal daily walked distance measured using a pedometer to predict maximum oxygen consumption per minute in patients undergoing lung resection. Eur J Cardiothorac Surg 2011, 39:756-62.

52. Knols R, Stappaerts K, Fransen J, et al: Isometric strength measurement for muscle weakness in cancer patients: reproducibility of isometric muscle strength measurements with a hand-held pull-gauge dynamometer in cancer patients. Support Care Canc 2002, 10:430-8.

53. Brown D, McMillan D, Milroy R: The correlation between fatigue, physical function, the systemic inflammatory response, and psychological distress in patients with advanced lung cancer. Cancer 2005, 103:377-82.

54. Maddocks M, Mockett S, Wilcock A: Is exercise an acceptable and practical therapy for people with or cured of cancer? A systematic review. Canc Treat Rev 2009, 35:383-390.

55. Jones $L$, Eves $N$, Haykowsky $M$, et al: Cardiorespiratory exercise testing in clinical oncology research: systematic review and practice recommendations. Lancet Oncol 2008, 9:757-765.

56. American Thoracic Society: ATS statement: guidelines for the six-minute walk test. Am J Respir Crit Care Med 2002, 166:111-117.

57. Alison JA, Kenny P, King MT, et al: Repeatability of the Six-Minute Walk Test and Relation to Physical Function in Survivors of a Critical Illness. Phys Ther 2012, 91(12):1556.

58. Solway S, Brooks D, Lacasse $Y$, et al: A qualitative systematic overview of the measurement properties of functional walk tests used in the cardiorespiratory domain. Chest 2001, 119:256-270.

59. Jenkins SC: 6-Minute walk test in patients with COPD: clinical applications in pulmonary rehabilitation. Physiotherapy 2007, 93:175-182.

60. Bellet N, Adams L, Morris N: Systematic review: The 6-minute walk test in outpatient cardiac rehabilitation: validity, reliability and responsiveness-a systematic review. Physiotherapy 2012, 98(4):277-287.

61. Sadaria K, Bahannon R: The 6-minute walk test: a brief review of literature. Clin Exerc Physiol 2001, 3:127.

62. Andersen $A$, Vinther $A$, Poulsen $L$, et al: Do patients with lung cancer benefit from physical exercise? Acta Oncol 2011, 50:307-13.

63. Maddocks M, Lewis M, Chauhan A, et al: Randomized controlled pilot study of neuromuscular electrical stimulation of the quadriceps in patients with non-small cell lung cancer. J Pain Symptom Manage 2009, 38:950-6.

64. Win T, Jackson A, Groves A, et al: Relationship of shuttle walk test and lung cancer surgical outcome. Eur J Cardiothorac Surg 2004, 26:1216-9.

65. Singh S, Morgan M, Scott S, et al: Development of a shuttle walking test of disability in patients with chronic airways obstruction. Thorax 1992, 47:1019-24.

66. Leenders N, Sherman W, Nagaraja H: Energy expenditure estimated by accelerometry and doubly labeled water: do they agree? Med Sci Sports Exerc 2006, 38:2165-2172.

67. Bluck L: Doubly labelled water for the measurement of total energy expenditure in man - progress and applications in the last decade. Br Nutr Foundation Nutr Bull 2008, 33:80-90.
68. Innes E: Handgrip strength testing: a review of the literature. Aust Occup Ther J 1999, 46:120-140.

69. Bohannon RW: Responsiveness of hand-held dynamometry to changes in limb muscle strength: a retrospective investigation of published research. Isokinet Exer Sci 2009, 17:221-5.

70. Martin $\mathrm{H}$ : Is hand-held dynamometry useful for the measurement of quadriceps strength in older people? A comparison with the gold standard biodex dynamometry. Gerontology 2006, 52:154-159.

71. Ciesla N, Dinglas V, Fan E, et al: Manual muscle testing: a method of measuring extremity muscle strength applied to critically ill patients. J Vis Exp 2011, 12(50):e2632.

72. Hough C, Lieu B, Caldwell E: Manual muscle strength testing of critically ill patients: feasibility and interobserver agreement. Crit Care 2011, 15:R43.

doi:10.1186/1471-2407-13-135

Cite this article as: Granger et al:: Functional capacity, physical activity and muscle strength assessment of individuals with non-small cell lung cancer: a systematic review of instruments and their measurement properties. BMC Cancer 2013 13:135.

\section{Submit your next manuscript to BioMed Central and take full advantage of:}

- Convenient online submission

- Thorough peer review

- No space constraints or color figure charges

- Immediate publication on acceptance

- Inclusion in PubMed, CAS, Scopus and Google Scholar

- Research which is freely available for redistribution
C) Biomed Central 